\title{
GLOBAL SOLUTIONS TO STOCHASTIC REACTION-DIFFUSION EQUATIONS WITH SUPER-LINEAR DRIFT AND MULTIPLICATIVE NOISE
}

\author{
By Robert C. DAlang ${ }^{1}$, Davar Khoshnevisan ${ }^{2}$ And Tusheng Zhang \\ EPF-Lausanne, University of Utah and University of Manchester
}

Let $\xi(t, x)$ denote space-time white noise and consider a reactiondiffusion equation of the form

$$
\dot{u}(t, x)=\frac{1}{2} u^{\prime \prime}(t, x)+b(u(t, x))+\sigma(u(t, x)) \xi(t, x),
$$

on $\mathbb{R}_{+} \times[0,1]$, with homogeneous Dirichlet boundary conditions and suitable initial data, in the case that there exists $\varepsilon>0$ such that $|b(z)| \geq$ $|z|(\log |z|)^{1+\varepsilon}$ for all sufficiently-large values of $|z|$. When $\sigma \equiv 0$, it is well known that such PDEs frequently have nontrivial stationary solutions. By contrast, Bonder and Groisman [Phys. D 238 (2009) 209-215] have recently shown that there is finite-time blowup when $\sigma$ is a nonzero constant. In this paper, we prove that the Bonder-Groisman condition is unimprovable by showing that the reaction-diffusion equation with noise is "typically" well posed when $|b(z)|=O\left(|z| \log _{+}|z|\right)$ as $|z| \rightarrow \infty$. We interpret the word "typically" in two essentially-different ways without altering the conclusions of our assertions.

1. Introduction. Let $\xi$ denote space-time white noise on $\mathbb{R}_{+} \times[0,1]$, and consider the parabolic stochastic partial differential equation

$$
\dot{u}(t, x)=\frac{1}{2} u^{\prime \prime}(t, x)+b(u(t, x))+\sigma(u(t, x)) \xi(t, x),
$$

$t>0, x \in(0,1)$, subject to the homogeneous Dirichlet boundary condition,

$$
u(t, 0)=u(t, 1)=0 \quad \text { for all } t>0,
$$

and the initial condition $u(0, \cdot)=u_{0}$ on $[0,1]$. Throughout, $\sigma, b$ and $u_{0}$ are assumed to be nonrandom and measurable real-valued functions on the real line.

Received June 2017; revised March 2018.

${ }^{1}$ Supported in part by the Swiss National Foundation for Scientific Research.

${ }^{2}$ Supported in part by NSF Grants DMS-1006903, DMS-1307470, DMS-1608575 and PHY1125915 through an NSF grant to the Kavli Institute for Theoretical Physics at UC Santa Barbara. Parts of this research were carried out while the authors were at the Banff International Research Station in April 2012.

MSC2010 subject classifications. Primary 60H15, 35K57; secondary 35R60, 35B45, 35B33.

Key words and phrases. Stochastic partial differential equations, reaction-diffusion equations, blow-up, logarithmic Sobolev inequality. 
It is well known that if, in addition, $b, \sigma$ are globally Lipschitz functions then any local solution of (1.1) is necessarily a global one. Note that the Lipshitz continuity of $\sigma$ and $b$ implies their sublinear growth; that is, $|b(z)|+|\sigma(z)|=O(|z|)$ as $|z| \rightarrow \infty$. In 2009, Bonder and Groisman [3] proved the following interesting complement.

THEOREM 1.1 (Bonder and Groisman [3]). Suppose, in addition, that $\sigma$ is a nonzero constant, $b \geq 0$ is convex and satisfies $\int_{1}^{\infty} \mathrm{d} z / b(z)<\infty$ and the initial function $u_{0}$ is nonnegative, continuous on $[0,1]$ and vanishes on $\{0,1\}$. Then there exists a random time $\tau$ such that $\mathrm{P}\{\tau<\infty\}=1$ and

$$
\lim _{t \uparrow \tau} \int_{0}^{1}|u(t, x)|^{2} \mathrm{~d} x=\infty \quad \text { almost surely. }
$$

REMARK 1.2. To be precise, Theorem 3.2 of Bonder and Groisman [3] implies the weaker conclusion that $\lim _{t \uparrow \tau} \sup _{x \in[0,1]}|u(t, x)|=\infty$ a.s. However, their proof yields the stronger result that

$$
\Phi(t):=\int_{0}^{1} u(t, x) \sin (\pi x) \mathrm{d} x
$$

explodes in finite time a.s.; see the discussion prior to the statement of Lemma 3.1 in [3]. This and the Cauchy-Schwarz inequality together yield Theorem 1.1.

Theorem 1.1 is surprising because, if we set $\sigma \equiv 0$, then the resulting reactiondiffusion equation (1.1) can have nontrivial global stationary solutions [3, 14, 26]. Therefore, we see that the introduction of any amount of additive spacetime white noise to a reaction diffusion equation removes the possibility of global well-posedness if the reaction term grows faster than a constant multiple of $|z|(\log |z|)^{1+\varepsilon}$ for some $\varepsilon>0$ (say) as either $z \rightarrow \infty$ or $z \rightarrow-\infty$.

Several papers in the literature discuss stochastic PDEs with locally Lipschitz coefficients that have polynomial growth and/or satisfy certain monotonicity conditions (see $[4,11,18,21,22]$, for instance). The typical example of such a coefficient is $b(u)=-u^{3}$, which has the effect of "pulling the solution back toward the origin." This is quite different from the situation that we discuss in this paper, where $b(u)$ will typically "push" the solution toward $\pm \infty$.

The goal of this article is to prove that the Bonder-Groisman theorem (Theorem 1.1) is optimal. In fact, we introduce two rather different methods that show that, under two different sets of natural conditions, if $|b(z)|=O(|z| \log |z|)$, then (1.1) is globally well posed. With this aim in mind, let us first introduce some notation.

DEFINITION 1.3. Suppose that $u_{0} \in L^{2}[0,1]$. We say that (1.1) has an $\mathbb{L}_{\text {loc }}^{2}$ solution $u$ if there exists a stopping time $\tau$ [with respect to the standard Brownian 
filtration generated by $\xi$; see Step 2, following (4.8) below] and an adapted continuous $L^{2}[0,1]$-valued random field $\{u(t, \cdot)\}_{t \in[0, \tau)}$ such that, almost surely on the event $\{\tau>t\}$,

$$
\begin{aligned}
\int_{0}^{1} u(t, x) \phi(x) \mathrm{d} x & \\
= & \int_{0}^{1} u_{0}(x) \phi(x) \mathrm{d} x+\frac{1}{2} \int_{(0, t) \times(0,1)} u(s, x) \phi^{\prime \prime}(x) \mathrm{d} s \mathrm{~d} x \\
& +\int_{(0, t) \times(0,1)} b(u(s, x)) \phi(x) \mathrm{d} s \mathrm{~d} x \\
& +\int_{(0, t) \times(0,1)} \sigma(u(s, x)) \phi(x) \xi(\mathrm{d} s \mathrm{~d} x),
\end{aligned}
$$

for every nonrandom test function $\phi \in C^{2}[0,1]$ that satisfies $\phi(0)=\phi(1)=0$.

Our first result can be stated as follows.

THEOREM 1.4. Suppose that $u_{0} \in L^{2}[0,1], \sigma: \mathbb{R} \rightarrow \mathbb{R}$ is bounded, and $|b(z)|=O(|z| \log |z|)$ as $|z| \rightarrow \infty$. Then every $\mathbb{L}_{\text {loc }}^{2}$-solution $u$ of (1.1) is a longtime solution; that is,

$$
\sup _{t \in[0, \tau \wedge T]} \int_{0}^{1}|u(t, x)|^{2} \mathrm{~d} x<\infty \quad \text { a.s. for every } T \in(0, \infty) .
$$

REMARK 1.5. Suppose $\tau$ is a maximal time up to which the solution can be constructed; that is,

$$
\sup _{t \in[0, \tau)}\|u(t)\|_{L^{2}[0,1]}=\infty \quad \text { a.s. }
$$

Then Theorem 1.4 implies that $\tau=\infty$ a.s. In this case, $\sup _{t \in[0, T]}\|u(t)\|_{L^{2}[0,1]}<$ $\infty$ a.s. for all $T>0$. The question of the existence of an $\mathbb{L}_{\text {loc }}^{2}$-solution of (1.1) under the assumptions of Theorem 1.4 is open.

Theorem 1.4 is an infinite-dimensional variation on aspects of the theory of Fang and Zhang [12] on stochastic differential equations with superlinear coefficients. We follow the Lyapunov function method of Fang and Zhang, and overcome the transition from finite to infinite dimensions by appealing to the sharp form of Gross' logarithmic Sobolev inequality for normalized Lebesgue measure [17]. We believe that our technique might also have other uses in infinite-dimensional stochastic analysis.

Our second result is based on an $L^{\infty}$ method and, as such, requires stronger regularity on the initial function $u_{0}$. In order to introduce it, we first need some notation. 
DEFINITION 1.6. For every $\alpha \in(0,1)$, we define $\mathbb{C}_{0}^{\alpha}$ to be the collection of all functions $f:[0,1] \rightarrow \mathbb{R}$ such that $f(0)=f(1)=0$ and

$$
\|f\|_{\mathbb{C}_{0}^{\alpha}}:=\sup _{0 \leq x<y \leq 1} \frac{|f(y)-f(x)|}{|y-x|^{\alpha}}<\infty .
$$

The space $\mathbb{C}_{0}^{1}$ will denote the collection of all Lipschitz-continuous functions $f$ : $[0,1] \rightarrow \mathbb{R}$ such that $f(0)=f(1)=0$. We sometimes write $\operatorname{Lip}(f)$ in place of $\|f\|_{\mathbb{C}_{0}^{1}}$.

In all cases, we see that $\mathbb{C}_{0}^{\alpha}$ is a Banach space endowed with the norm $\|\cdot\|_{\mathbb{C}_{0}^{\alpha}}$. Let us also recall the following definition (see Dalang [8] or [7]).

DEFINITION 1.7. A random field solution to (1.1) is a jointly measurable and adapted space-time process $u:=\{u(t, x)\}_{(t, x) \in \mathbb{R}_{+} \times[0,1]}$ such that, for all $(t, x) \in$ $\mathbb{R}_{+} \times[0,1]$

$$
\begin{aligned}
u(t, x)= & \left(\mathcal{G}_{t} u_{0}\right)(x)+\int_{(0, t) \times(0,1)} G_{t-s}(x, y) b(u(s, y)) \mathrm{d} s \mathrm{~d} y \\
& +\int_{(0, t) \times(0,1)} G_{t-s}(x, y) \sigma(u(s, y)) \xi(\mathrm{d} s \mathrm{~d} y),
\end{aligned}
$$

a.s., where $\left\{\mathcal{G}_{t}\right\}_{t \geq 0}$ and $G$ are respectively the heat semigroup and heat kernel for the Dirichlet Laplacian, and are recalled in (2.4) and (2.5) below.

REMARK 1.8. The stochastic integral in Definition 1.7 is not always defined in the sense of Walsh [28] since the Walsh integral is defined provided that for all $t>0$ and $x \in[0,1]$,

$$
\mathrm{E}\left[\int_{(0, t) \times(0,1)}\left[G_{t-s}(x, y) \sigma(u(s, y))\right]^{2} \mathrm{~d} s \mathrm{~d} y\right]<\infty .
$$

Instead, we are using a localized version of the Walsh integral, for whose existence we require only that for all $t>0$ and $x \in[0,1]$,

$$
\int_{(0, t) \times(0,1)}\left[G_{t-s}(x, y) \sigma(u(s, y))\right]^{2} \mathrm{~d} s \mathrm{~d} y<\infty \quad \text { a.s. }
$$

We are now in position to present our second complement to the BonderGroisman theorem (Theorem 1.1).

THEOREM 1.9. Suppose that:

(i) $u_{0} \in \bigcup_{0<\alpha \leq 1} \mathbb{C}_{0}^{\alpha}$;

(ii) $b$ and $\sigma$ are locally Lipschitz functions such that

$$
|b(z)|=O(|z| \log |z|) \text { and }|\sigma(z)|=o\left(|z|(\log |z|)^{1 / 4}\right),
$$

as $|z| \rightarrow \infty$. 
Then the SPDE (1.1) has a random field solution $u$ in $C\left(\mathbb{R}_{+} \times[0,1]\right)$, and this solution is unique. In particular, $u$ satisfies

$$
\sup _{t \in[0, T]} \sup _{x \in[0,1]}|u(t, x)|<\infty \quad \text { a.s. for all } T \in(0, \infty) .
$$

Recall that (1.2) means that there is a constant $C<\infty$ such that for $|z|$ large enough, $|b(z)| \leq C|z| \log |z|$, and $\lim _{|z| \rightarrow \infty}\left|\sigma(z) /\left(z(\log |z|)^{1 / 4}\right)\right|=0$.

We conclude the Introduction with a few words about the notation that is used consistently in this paper.

\section{Remarks on notation.}

1. Throughout the paper, we write $\mathbb{L}^{p}$ in place of $L^{p}[0,1]$ for every $1 \leq p \leq \infty$. In particular, $\|f\|_{\mathbb{L}^{\infty}}$ and $\|f\|_{\mathbb{L}^{2}}$, respectively, denote the essential supremum and the $\mathbb{L}^{2}$-norm of a suitable function $f:[0,1] \rightarrow \mathbb{R}$.

2. Throughout, we define $\log _{+}(w):=\log (\max (w, \mathrm{e}))$ for all $w \in \mathbb{R}$.

3. If $f$ and $g$ are nonnegative functions on some space $X$, then we write $f(x) \lesssim$ $g(x)$ for all $x \in X$ [equivalently, $g(x) \gtrsim f(x)$ for all $x \in X$ ] to mean that there exists a finite constant $A$ such that $f(x) \leq A g(x)$ for all $x \in X$.

4. If $f$ and $g$ are nonnegative functions on some space $X$, then we write $f(x) \asymp$ $g(x)$ for all $x \in X$ to mean that $f(x) \lesssim g(x) \lesssim f(x)$ for all $x \in X$.

2. Proof of Theorem 1.4. We will appeal to the logarithmic Sobolev inequality of Gross [17] in the following form: For every $\varepsilon \in(0,1)$ and differentiable function $h:[0,1] \rightarrow \mathbb{R}$ that vanishes continuously on $\{0,1\}$,

$$
\begin{aligned}
& \int_{0}^{1}|h(x)|^{2} \log |h(x)| \mathrm{d} x \\
& \quad \leq \varepsilon\left\|h^{\prime}\right\|_{\mathbb{L}^{2}}^{2}+\frac{1}{4} \log (1 / \varepsilon)\|h\|_{\mathbb{L}^{2}}^{2}+\|h\|_{\mathbb{L}^{2}}^{2} \log \left(\|h\|_{\mathbb{L}^{2}}^{2}\right),
\end{aligned}
$$

where $0 \log 0:=0$. One can derive this logarithmic Sobolev inequality from formula (5.4) in ref. [17] using the fact that

$$
\log \left(\left\|\mathcal{G}_{\varepsilon}\right\|_{2 \rightarrow \infty}\right) \leq \frac{1}{2} \sup _{x \in[0,1]} \log \left(\int_{0}^{1}\left|G_{\varepsilon}(x, y)\right|^{2} \mathrm{~d} y\right) \leq \frac{1}{4} \log (1 / \varepsilon),
$$

where $\mathcal{G}:=\left\{\mathcal{G}_{t}\right\}_{t \geq 0}$ denotes the heat semigroup and $G:(0, \infty) \times[0,1]^{2} \rightarrow \mathbb{R}_{+}$ the corresponding heat kernel; see (2.5) below.

Let $\mathbb{L}^{2} \log \mathbb{L}$ denote the vector space of all measurable functions $h:[0,1] \rightarrow \mathbb{R}$ that satisfy

$$
\|h\|_{\mathbb{L}^{2} \log \mathbb{L}}:=\left(\int_{0}^{1}|h(x)|^{2} \log _{+}|h(x)| \mathrm{d} x\right)^{1 / 2}<\infty
$$


Now, $\int_{0}^{1}|h(x)|^{2} \mathbb{1}_{\{|h(x)|<\mathrm{e}\}} \mathrm{d} x \leq\|h\|_{\mathbb{L}^{2}}^{2}$ and

$$
\begin{aligned}
& \int_{0}^{1}|h(x)|^{2}|\log | h(x)|| \mathbb{1}_{\{0<|h(x)| \leq 1\}} \mathrm{d} x \\
& \quad \leq \int_{0}^{1}|h(x)| \log (1 /|h(x)|) \mathbb{1}_{\{0<|h(x)| \leq 1\}} \mathrm{d} x \leq \mathrm{e}^{-1},
\end{aligned}
$$

since $y \log (1 / y) \leq \mathrm{e}^{-1}$ for all $y \in[0,1]$. Therefore,

$$
\begin{aligned}
\|h\|_{\mathbb{L}^{2} \log \mathbb{L}}= & \int_{0}^{1}|h(x)|^{2} \mathbb{1}_{\{|h(x)| \leq \mathrm{e}\}} \mathrm{d} x+\int_{0}^{1}|h(x)|^{2} \log |h(x)| \mathbb{1}_{\{|h(x)|>\mathrm{e}\}} \mathrm{d} x \\
\leq & \|h\|_{\mathbb{L}^{2}}+\int_{0}^{1}|h(x)|^{2} \log |h(x)| \mathrm{d} x \\
& -\int_{0}^{1}|h(x)|^{2} \log |h(x)| \mathbb{1}_{\{0<|h(x)|<1\}} \mathrm{d} x \\
& -\int_{0}^{1}|h(x)|^{2} \log |h(x)| \mathbb{1}_{\{1 \leq|h(x)| \leq \mathrm{e}\}} \mathrm{d} x \\
\leq & \|h\|_{\mathbb{L}^{2}}+\int_{0}^{1}|h(x)|^{2} \log |h(x)| \mathrm{d} x+\mathrm{e}^{-1} .
\end{aligned}
$$

Together with these remarks, and a standard density argument, Gross' logarithmic Sobolev inequality can be cast in the following manner in terms of $\mathbb{L}^{2} \log \mathbb{L}$ norms.

THEOREM 2.1 (The logarithmic Sobolev inequality). If $h \in W^{1,2}[0,1]$ vanishes continuously at the boundary, then

$$
\|h\|_{\mathbb{L}^{2} \log \mathbb{L}}^{2} \leq \varepsilon\left\|h^{\prime}\right\|_{\mathbb{L}^{2}}^{2}+K_{\varepsilon}\|h\|_{\mathbb{L}^{2}}^{2}+\|h\|_{\mathbb{L}^{2}}^{2} \log _{+}\left(\|h\|_{\mathbb{L}^{2}}^{2}\right)+\mathrm{e}^{-1},
$$

for every $\varepsilon \in(0,1)$, where $K_{\varepsilon}:=1+\frac{1}{4} \log (1 / \varepsilon)$.

We are ready to verify Theorem 1.4.

Proof OF THEOREM 1.4. For every $R>0$, consider the stopping times

$$
\tau(R):= \begin{cases}\inf \left\{t \in[0, \tau):\|u(t)\|_{\mathbb{L}^{2}}>R\right\} & \text { if }\{\ldots\} \neq \varnothing, \\ \tau & \text { otherwise. }\end{cases}
$$

We aim to prove that $\mathrm{P}\left\{\sup _{t<\tau \wedge T}\|u(t)\|_{\mathbb{L}^{2}}=\infty\right\}=0$.

Since

$$
\left\{\sup _{t<\tau \wedge T}\|u(t)\|_{\mathbb{L}^{2}}=\infty\right\}=\bigcap_{R>0}\{\tau(R)<\tau \wedge T\},
$$

it suffices to prove that

$$
\lim _{R \rightarrow \infty} \mathrm{P}\{\tau(R)<\tau \wedge T\}=0 \quad \text { for all } T>0 .
$$


For every constant $R>0$, consider the following stochastic PDE with random forcing and no reaction term:

$$
\dot{v}_{R}(t, x)=\frac{1}{2} v_{R}^{\prime \prime}(t, x)+\sigma(u(t \wedge \tau(R), x)) \xi(t, x) \quad[t>0,0 \leq x \leq 1] .
$$

We consider (2.2) subject to the initial condition $v_{R}(0)=0$ and the same boundary conditions as $(1.1)$; that is, $v_{R}(t, 0)=v_{R}(t, 1)=0$ for all $t>0$. The solution process $t \mapsto v_{R}(t)$ exists, is unique [in $\mathbb{L}^{2}$ ] and is an $\mathbb{L}^{2}$-valued stochastic process that satisfies the following weak random integral equation viewed as an equation in $\mathbb{L}^{2}$ :

$$
v_{R}(t, x)=\int_{(0, t) \times(0,1)} G_{t-s}(x, y) \sigma(u(s \wedge \tau(R), y)) \xi(\mathrm{d} s \mathrm{~d} y) .
$$

Notice that the stochastic integral is well defined as a Walsh integral because $\sigma$ is assumed to be bounded.

In the above, the function $G:(0, \infty) \times[0,1]^{2} \rightarrow \mathbb{R}_{+}$denotes the heat kernel; as was mentioned earlier, we will use $\mathcal{G}:=\left\{\mathcal{G}_{t}\right\}_{t \geq 0}$ to denote the corresponding heat semigroup. That is, $\mathcal{G}_{0} f=f$, for $t>0$,

$$
\left(\mathcal{G}_{t} f\right)(x):=\int_{0}^{1} G_{t}(x, y) f(y) \mathrm{d} y,
$$

and $(t, x, y) \mapsto G_{t}(x, y)$ denotes the fundamental solution to the heat equation $\dot{G}=\frac{1}{2} G^{\prime \prime}$ on $(0, \infty) \times[0,1]$ with zero boundary conditions, namely

$$
G_{t}(x, y)=2 \sum_{n=1}^{\infty} \sin (n \pi x) \sin (n \pi y) \exp \left(-\frac{n^{2} \pi^{2} t}{2}\right),
$$

for every $t>0$ and $x, y \in[0,1]$. We recall the well-known inequality, valid for all $t>0$ and $x, y \in[0,1]$ :

$$
0 \leq G_{t}(x, y) \leq p(t, x-y),
$$

where $p(t, \cdot)$ denotes the standard $N(0, t)$ probability density function. The preceding assertion is an immediate consequence of the classical fact that the mapping $(0, \infty) \times[0,1]^{2} \ni(s, a, b) \mapsto G_{s}(a, b)$ describes the transition densities of a Brownian motion killed upon reaching $\{0,1\}$; see Bass [2], Chapter 2, Section 7.

Define, for every fixed $R>0$,

$$
d_{R}:=u-v_{R}
$$

We may observe that $d_{R}$ is an $\mathbb{L}_{\text {loc }}^{2}$-solution of the following heat equation: On $\{\tau>t\}$

$$
\dot{d}_{R}(t)=\frac{1}{2} d_{R}^{\prime \prime}(t)+b\left(v_{R}(t)+d_{R}(t)\right)
$$


subject to initial condition $d_{R}(0)=u_{0}$ and boundary values $d_{R}(t, 0)=d_{R}(t, 1)=$ 0 for all $t>0$. It should be emphasized that (2.7) is an ordinary partial differential equation with a random coefficient.

Choose and fix some $T>0$. Since $\sigma$ is a bounded measurable function, standard estimates [28], Chapter 3, show that there exists $\beta>0$ such that for all $p \in(2, \infty)$, $x, y \in[0,1]$, and $s, t \in[0, T]$,

$$
\mathrm{E}\left(\left|v_{R}(t, x)-v_{R}(s, y)\right|^{p}\right) \lesssim|t-s|^{p \beta}+|x-y|^{p \beta},
$$

where the implied constant depends only on $p$ and $T$. Since $v_{R}(0)$ vanishes on $\{0,1\}$, a standard application of the Kolmogorov continuity theorem for random fields [20], p. 31, then shows that

$$
A_{T}:=\sup _{R>0} \mathrm{E}\left(\sup _{t \in[0, T]} \sup _{x \in[0,1]}\left|v_{R}(t, x)\right|\right)<\infty .
$$

Consider the stopping time

$$
\tau_{M}(R):=\inf \left\{t>0: \sup _{x \in[0,1]}\left|v_{R}(t, x)\right|>M\right\} \quad \text { for every } M>0 .
$$

It follows from (2.9) and the Chebyshev inequality that

$$
\sup _{R>0} \mathrm{P}\left\{\tau_{M}(R)<T\right\} \leq \frac{A_{T}}{M} .
$$

Next, we observe that (2.8) and a suitable form of the Kolmogorov continuity theorem [20], p. 31, together show also that $v_{R}(\cdot, \cdot)$ has a version with continuous sample paths a.s. The same Kolmogorov continuity theorem also shows that the process $u(\cdot \wedge \tau(R))(\cdot)$ has a jointly continuous version (for $t>0$ and $x \in[0,1]$ ). Indeed, from the "weak" formulation of Definition 1.7, one deduces as in [28], Chapter III, that $u(t)$ is also a mild solution of (1.1), which is $L^{2}$-bounded prior to time $\tau(R)$, so using the growth condition on $b$ and the fact that $\sigma$ is bounded, one easily checks the conditions of the Kolmogorov continuity theorem to deduce the existence of a jointly continuous version (for $t>0, x \in[0,1])$ of $u(\cdot \wedge \tau(R))(\cdot)$.

Define two random space-time functions $D$ and $V$ as

$$
D(t):=d_{R}\left(t \wedge \tau(R) \wedge \tau_{M}(R)\right), \quad V(t):=v_{R}\left(t \wedge \tau(R) \wedge \tau_{M}(R)\right) \quad[0 \leq t \leq T],
$$

all the time suppressing the dependence of $D$ and $V$ on $(R, M)$, as well as on the spatial variable $x \in[0,1]$.

In order to show that for $s>0, D(s)$ has some regularity as a function of $x$, let $H_{0}^{1}$ denote the completion of $C_{0}^{\infty}(0,1)$ under the Sobolev norm $\|u\|_{H_{0}^{1}}:=$ $\left\{\int_{0}^{1}\left|u^{\prime}(x)\right|^{2} \mathrm{~d} x\right\}^{1 / 2}$. We claim that for $t>0, D(t) \in H_{0}^{1}$, that is,

$$
D^{\prime}(t) \in \mathbb{L}^{2} \quad \text { a.s., for all } t>0 .
$$

In order to verify (2.11), recall that

$$
\left\|\mathcal{G}_{t} f\right\|_{H_{0}^{1}} \leq t^{-1 / 2}\|f\|_{\mathbb{L}^{2}} \quad \text { for every } f \in \mathbb{L}^{2}
$$


see, for example, Cerrai [5], (2.6). Moreover, [5], (2.7),

(2.13) $\left\|\mathcal{G}_{t} f\right\|_{\mathbb{L}^{2}} \leq c_{p} t^{-(2-p) /(4 p)}\|f\|_{\mathbb{L}^{p}} \quad$ for every $f \in \mathbb{L}^{p}$ and $p \in(1,2]$.

The $L \log L$ growth of $b$ implies that for all $p \in(1,2)$,

$$
\|b(V(s)+D(s))\|_{\mathbb{L}^{p}} \leq C_{p, M}\left(1+\|D(s)\|_{\mathbb{L}^{2}}\right) \quad \text { for all } s>0,
$$

where $C_{p, M}$ is a finite constant.

The mild formulation of (2.7) yields

$$
D(t)=\mathcal{G}_{t} u_{0}+\int_{0}^{t} \mathcal{G}_{t-s}(b(V(s)+D(s))) \mathrm{d} s \quad[t>0]
$$

Therefore,

$$
\|D(t)\|_{H_{0}^{1}} \leq\left\|\mathcal{G}_{t} u_{0}\right\|_{H_{0}^{1}}+\int_{0}^{t}\left\|\mathcal{G}_{t-s}(b(V(s)+D(s)))\right\|_{H_{0}^{1}} \mathrm{~d} s .
$$

By (2.12) and the semigroup property of $t \mapsto \mathcal{G}_{t}$, the right-hand side is bounded above by

$$
t^{-1 / 2}\left\|u_{0}\right\|_{\mathbb{L}^{2}}+\int_{0}^{t}\left(\frac{2}{t-s}\right)^{1 / 2}\left\|\mathcal{G}_{(t-s) / 2}(b(V(s)+D(s)))\right\|_{\mathbb{L}^{2}} \mathrm{~d} s .
$$

By (2.13), then (2.14), for every $p \in(1,2)$ and $t>0$, this is bounded above by

$$
\begin{aligned}
t^{-1 / 2}\left\|u_{0}\right\|_{\mathbb{L}^{2}}+c_{p} \int_{0}^{t}\left(\frac{2}{t-s}\right)^{1 / 2}\left(\frac{2}{t-s}\right)^{(2-p) /(4 p)}\|b(V(s)+D(s))\|_{\mathbb{L}^{p}} \mathrm{~d} s \\
\leq t^{-1 / 2}\left\|u_{0}\right\|_{\mathbb{L}^{2}} \\
\quad+c_{p} C_{p, M}\left(1+\sup _{0 \leq s \leq t}\|D(s)\|_{\mathbb{L}^{2}}\right) \cdot \int_{0}^{t}\left(\frac{2}{t-s}\right)^{(2+p) /(4 p)} \mathrm{d} s,
\end{aligned}
$$

which is finite since $(2+p) /(4 p)<1$. This implies $(2.11)$.

Now that we have proved (2.11), we may combine (2.7) with the chain rule of [25], Lemma 1.1, in order to see that for every $t \in[0, T]$,

$$
\begin{aligned}
\|D(t)\|_{\mathbb{L}^{2}}^{2}= & \left\|u_{0}\right\|_{\mathbb{L}^{2}}^{2}+2 \int_{0}^{t}\langle\dot{D}(s), D(s)\rangle_{\mathbb{L}^{2}} \mathrm{~d} s \\
= & \left\|u_{0}\right\|_{\mathbb{L}^{2}}^{2}+2 \int_{0}^{t}\left\langle\frac{1}{2} D^{\prime \prime}(s), D(s)\right\rangle_{\mathbb{L}^{2}} \mathrm{~d} s \\
& +2 \int_{0}^{t}\langle b(V(s)+D(s)), D(s)\rangle_{\mathbb{L}^{2}} \mathrm{~d} s \\
= & \left\|u_{0}\right\|_{\mathbb{L}^{2}}^{2}-\int_{0}^{t}\left\|D^{\prime}(s)\right\|_{\mathbb{L}^{2}}^{2} \mathrm{~d} s \\
& +2 \int_{0}^{t}\langle b(V(s)+D(s)), D(s)\rangle_{\mathbb{L}^{2}} \mathrm{~d} s,
\end{aligned}
$$


thanks to integration by parts [in fact, the second equality is formal, since $D^{\prime \prime}(s)$ may not belong to $\mathbb{L}^{2}$ ]; the equality of the third line with the first is obtained by smoothing the term $b\left(v_{R}(t)+d_{R}(t)\right)$ in (2.7) and passing to the limit using the continuity property in [25], Lemma 1.2).

As in [12], we consider the Lyapunov function,

$$
\Phi(r):=\exp \left(\int_{0}^{r} \frac{\mathrm{d} z}{1+z \log _{+} z}\right) \quad[r>0] .
$$

Owing to (2.16) and a second application of the chain rule,

$$
\begin{aligned}
\Phi\left(\|D(t)\|_{\mathbb{L}^{2}}^{2}\right)= & \Phi\left(\left\|u_{0}\right\|_{\mathbb{L}^{2}}^{2}\right)-\int_{0}^{t} \Phi^{\prime}\left(\|D(s)\|_{\mathbb{L}^{2}}^{2}\right)\left\|D^{\prime}(s)\right\|_{\mathbb{L}^{2}}^{2} \mathrm{~d} s \\
& +2 \int_{0}^{t} \Phi^{\prime}\left(\|D(s)\|_{\mathbb{L}^{2}}^{2}\langle b(V(s)+D(s)), D(s)\rangle_{\mathbb{L}^{2}} \mathrm{~d} s .\right.
\end{aligned}
$$

Define

$$
C_{b}:=\sup _{z \in \mathbb{R}} \frac{|b(z)|}{1+|z| \log _{+}|z|},
$$

and observe that $C_{b} \in(0, \infty)$, thanks to the $L \log L$ growth of $b$. Moreover,

$$
\begin{aligned}
\langle b(V(s) & +D(s)),\left.D(s)\right|_{\mathbb{L}^{2}} \\
\leq & C_{b} \int_{0}^{1}\left[1+\{|V(s, x)|+|D(s, x)|\} \log _{+}(|V(s, x)|+|D(s, x)|)\right] \\
& \times|D(s, x)| \mathrm{d} x \\
\leq & C_{b} \int_{0}^{1}\left[1+\{M+|D(s, x)|\} \log _{+}(M+|D(s, x)|)\right] \times|D(s, x)| \mathrm{d} x,
\end{aligned}
$$

for every $s \in[0, T]$. In particular,

$$
\langle b(V(s)+D(s)), D(s)\rangle_{\mathbb{L}^{2}} \lesssim\|D(s)\|_{\mathbb{L}^{2} \log \mathbb{L}}^{2}+\|D(s)\|_{\mathbb{L}^{2}}^{2}+\|D(s)\|_{\mathbb{L}^{1}},
$$

for all $s \in[0, T]$, where the implied constant depends only on $\left(C_{b}, M\right)$. The Cauchy-Schwarz inequality yields

$$
\|D(s)\|_{\mathbb{L}^{1}} \leq\|D(s)\|_{\mathbb{L}^{2}} \lesssim\|D(s)\|_{\mathbb{L}^{2}}^{2}+1 \quad \text { for all } s>0,
$$

and hence

$$
\langle b(V(s)+D(s)), D(s)\rangle_{\mathbb{L}^{2}} \leq \bar{C}\left\{\|D(s)\|_{\mathbb{L}^{2} \log \mathbb{L}}^{2}+\|D(s)\|_{\mathbb{L}^{2}}^{2}+1\right\},
$$

uniformly for all $s \in[0, T]$, where $\bar{C}$ is a nonrandom and finite constant that depends only on $\left(C_{b}, M\right)$. Recall that $D$ is differentiable in $x, D^{\prime}(s) \in \mathbb{L}^{2}$ a.s. for all $s>0$ and $D$ satisfies the Dirichlet boundary condition. Therefore, we may apply 
the logarithmic Sobolev inequality [Theorem 2.1] with $\varepsilon:=1 /(2 \bar{C})$ in order to see that

$$
\begin{aligned}
& \langle b(V(s)+D(s)), D(s)\rangle_{\mathbb{L}^{2}} \\
& \quad \leq \frac{1}{2}\left\|D^{\prime}(s)\right\|_{\mathbb{L}^{2}}^{2}+c_{*}\left\{\|D(s)\|_{\mathbb{L}^{2}}^{2}+\|D(s)\|_{\mathbb{L}^{2}}^{2} \log _{+}\left(\|D(s)\|_{\mathbb{L}^{2}}^{2}\right)+1\right\},
\end{aligned}
$$

uniformly for all $s \in[0, T]$, where $c_{*}$ is a nonrandom and finite constant, and depends only on $\left(C_{b}, M\right)$. Since $a \log _{+} a+1 \geq a$ for all $a \geq 0$ and because the coefficient of $\left\|D^{\prime}(s)\right\|_{\mathbb{L}^{2}}^{2}$ is one-half in the above displayed inequality, we can deduce the following from (2.17):

$$
\begin{aligned}
& \Phi\left(\|D(t)\|_{\mathbb{L}^{2}}^{2}\right) \\
& \quad \lesssim \Phi\left(\left\|u_{0}\right\|_{\mathbb{L}^{2}}^{2}\right)+\int_{0}^{t} \Phi^{\prime}\left(\|D(s)\|_{\mathbb{L}^{2}}^{2}\right)\left\{1+\|D(s)\|_{\mathbb{L}^{2}}^{2} \log _{+}\left(\|D(s)\|_{\mathbb{L}^{2}}^{2}\right)\right\} \mathrm{d} s,
\end{aligned}
$$

uniformly for all $t \in[0, T]$, where the implied constant is nonrandom and finite, and depends only on $\left(C_{b}, M\right)$. But $\Phi^{\prime}(r)\left[1+r \log _{+} r\right]=\Phi(r)$ for all $r \geq 0$. Therefore, the preceding inequality implies that

$$
\Phi\left(\|D(t)\|_{\mathbb{L}^{2}}^{2}\right) \lesssim \Phi\left(\left\|u_{0}\right\|_{\mathbb{L}^{2}}^{2}\right)+\int_{0}^{t} \Phi\left(\|D(s)\|_{\mathbb{L}^{2}}^{2}\right) \mathrm{d} s,
$$

uniformly for all $t \in[0, T]$, where the implied constant is nonrandom and finite, and depends only on $\left(C_{b}, M, T\right)$, but not on $R$. Thanks to the definitions of $\tau(R)$ and $\tau_{M}(R)$, we know already that $\|D(t)\|_{\mathbb{L}^{2}} \leq R+M<\infty$ for all $t \in[0, T]$. It then follows from (2.18) and Gronwall's inequality that $\sup _{t \in[0, T]} \Phi\left(\|D(t)\|_{\mathbb{L}^{2}}^{2}\right)$ is a.s. bounded from above by a nonrandom finite number $B\left(C_{b}, M, T\right)$, that depends only on $\left(C_{b}, M, T\right)$ (but not on $R$ ), whence

$$
\sup _{R>0} \mathrm{E}\left[\Phi\left(\left\|d_{R}\left(T \wedge \tau(R) \wedge \tau_{M}(R)\right)\right\|_{\mathbb{L}^{2}}^{2}\right)\right] \leq B\left(C_{b}, M, T\right)
$$

Next, we observe that, almost surely on the event $\left\{\tau(R)<\tau \wedge T \leq \tau_{M}(R)\right\}$,

$$
\begin{aligned}
\left\|d_{R}\left(T \wedge \tau(R) \wedge \tau_{M}(R)\right)\right\|_{\mathbb{L}^{2}} & =\left\|d_{R}(\tau(R))\right\|_{\mathbb{L}^{2}} \\
& =\left\|u(\tau(R))-v_{R}(\tau(R))\right\|_{\mathbb{L}^{2}} \\
& \geq\|u(\tau(R))\|_{\mathbb{L}^{2}-\left\|v_{R}(\tau(R))\right\|_{\mathbb{L}^{2}}} \\
& =R-\left\|v_{R}(\tau(R))\right\|_{\mathbb{L}^{2}} .
\end{aligned}
$$

On the other hand,

$$
\begin{aligned}
\left\|v_{R}(\tau(R))\right\|_{\mathbb{L}^{2}} & \leq \sup _{t \in[0, \tau \wedge T)} \sup _{x \in[0,1]}\left|v_{R}(t, x)\right| \\
& \leq M \quad \text { a.s. on }\left\{\tau(R)<\tau \wedge T \leq \tau_{M}(R)\right\} .
\end{aligned}
$$


Thus, we see that

$$
\left\|d_{R}\left(T \wedge \tau(R) \wedge \tau_{M}(R)\right)\right\|_{\mathbb{L}^{2}} \geq R-M \quad \text { a.s. on }\left\{\tau(R)<\tau \wedge T \leq \tau_{M}(R)\right\},
$$

whence

$$
\begin{aligned}
& \Phi\left(\left\|d\left(T \wedge \tau(R) \wedge \tau_{M}(R)\right)\right\|_{\mathbb{L}^{2}}^{2}\right) \\
& \quad \geq \Phi\left((R-M)^{2}\right) \quad \text { a.s. on }\left\{\tau(R)<\tau \wedge T \leq \tau_{M}(R)\right\},
\end{aligned}
$$

as long as $R>M$. Combine this with (2.19) to see that

$$
\mathrm{P}\left\{\tau(R)<\tau \wedge T \leq \tau_{M}(R)\right\} \leq \frac{B\left(C_{b}, M, T\right)}{\Phi\left((R-M)^{2}\right)} \quad \text { for all } R>M>0 .
$$

The preceding inequality and (2.10) together show that

$$
\mathrm{P}\{\tau(R)<\tau \wedge T\} \leq \frac{B\left(C_{b}, M, T\right)}{\Phi\left((R-M)^{2}\right)}+\frac{A_{T}}{M},
$$

for all $R>M$. We first let $R \rightarrow \infty$ and then $M \rightarrow \infty$ in order to deduce (2.1). This completes the proof of Theorem 1.4.

3. Prelude to the proof of Theorem 1.9. Throughout this section, we consider (1.1) only in the classical case where

$b$ and $\sigma$ are globally Lipschitz continuous.

It is well known that, in this case, (1.1) is well posed (see Walsh [28], Chapter 3). Here, we develop some a priori moment bounds. One of our main goals is to establish a priori smoothness bounds for the solution of (1.1) that are valid up to and including the boundary of $[0,1]$. This endeavor requires some careful estimates and ultimately leads to an interesting optimal regularity theorem [Theorem 3.4] that forms one of the main ingredients in the proof of Theorem 1.9.

3.1. Moment bounds. We begin by establishing moment bounds for the solution $u$ to (1.1). With this goal in mind, we frequently use the elementary fact that for every globally Lipschitz function $f: \mathbb{R} \rightarrow \mathbb{R}$, there are constants $c(f)$ and $\mathrm{L}(f)$ such that

$$
|f(z)| \leq c(f)+\mathrm{L}(f)|z| \quad \text { for all } z \in \mathbb{R} .
$$

One possibility is to take $c(f)=|f(0)|$ and $\mathrm{L}(f)=\operatorname{Lip}(f)$, but often, $\mathrm{L}(f)$ can be chosen strictly smaller than $\operatorname{Lip}(f)$. We will only consider the case where

$$
\mathrm{L}(b) \geq 4 \mathrm{~L}(\sigma)^{4}>0 .
$$

The significance of this assumption, which is not a restriction since $\mathrm{L}(b)$ can be chosen arbitrarily large, will manifest itself later on in the proof of Theorem 1.9.

Throughout this section, (3.2) will be assumed tacitly. The next statement, which gives a bound on $k$ th moments of $u(t, x)$ for small $k$, is a refinement of a result that appears in [19], Theorem 5.5. 
Proposition 3.1. There exists a finite universal constant A such that

$$
\sup _{x \in[0,1]} \mathrm{E}\left(|u(t, x)|^{k}\right) \leq A^{k}\left(\left\|u_{0}\right\|_{\mathbb{L}^{\infty}}+\frac{c(b)}{L(b)}+\frac{c(\sigma)}{\sqrt{L(\sigma)}}\right)^{k} \cdot \exp (A k L(b) t),
$$

uniformly for all real numbers $t \geq 0$ and $k \in\left[2, \sqrt{L(b)} / L(\sigma)^{2}\right]$.

REMARK 3.2. Condition (3.2) ensures that the interval $\left[2, \sqrt{\mathrm{L}(b)} / \mathrm{L}(\sigma)^{2}\right]$ is nonempty.

ProOF. Throughout, we write $\mathcal{B}(t)$ for the box

$$
\mathcal{B}(t):=(0, t) \times(0,1), \quad \text { for every } t \geq 0 .
$$

In light of Definition 1.7,

$$
\begin{aligned}
u(t, x)= & \left(\mathcal{G}_{t} u_{0}\right)(x)+\int_{\mathcal{B}(t)} G_{t-s}(x, y) b(u(s, y)) \mathrm{d} s \mathrm{~d} y \\
& +\int_{\mathcal{B}(t)} G_{t-s}(x, y) \sigma(u(s, y)) \xi(\mathrm{d} s \mathrm{~d} y),
\end{aligned}
$$

where $\mathcal{G}$ and $G$ were defined respectively in (2.4) and (2.5). The solution $u$ to (1.1) has a [jointly] continuous version which is the unique continuous solution of (3.4).

Let us also recall that one verifies the existence of a solution to (3.4) by applying Picard's iteration method as follows: Set $u_{0}(t, x):=u_{0}(x)$ for all $x \in[0,1]$, and then iteratively define

$$
\begin{aligned}
u_{n+1}(t, x):= & \left(\mathcal{G}_{t} u_{0}\right)(x)+\int_{\mathcal{B}(t)} G_{t-s}(x, y) b\left(u_{n}(s, y)\right) \mathrm{d} s \mathrm{~d} y \\
& +\int_{\mathcal{B}(t)} G_{t-s}(x, y) \sigma\left(u_{n}(s, y)\right) \xi(\mathrm{d} s \mathrm{~d} y) .
\end{aligned}
$$

Then

(3.5) $\lim _{n \rightarrow \infty} u_{n}(t, x)=u(t, x) \quad$ in $L^{k}(\Omega)$ for all $k \geq 1, t \geq 0$, and $x \in[0,1]$;

see Walsh [28], Chapter 3.

We now follow Foondun and Khoshnevisan [13] and consider a two-parameter family $\left\{\mathcal{N}_{\beta, k}\right\}_{\beta>0, k \geq 1}$ of norms-each defined on the space of space-time random fields - as follows: For all real numbers $\beta>0$ and $k \geq 1$, and for every space-time random field $\Phi:=\{\Phi(t, x) ; t \geq 0, x \in[0,1]\}$,

$$
\mathcal{N}_{\beta, k}(\Phi):=\sup _{t \geq 0} \sup _{x \in[0,1]}\left(\mathrm{e}^{-\beta t}\|\Phi(t, x)\|_{k}\right) \text {. }
$$

Since $\left|\left(\mathcal{G}_{t} u_{0}\right)(x)\right| \leq\left\|u_{0}\right\|_{\mathbb{L}^{\infty}}$ for all $x \in[0,1]$ and $t>0$, we can write

$$
\mathcal{N}_{\beta, k}\left(u_{n+1}\right) \leq\left\|u_{0}\right\|_{\mathbb{L}^{\infty}}+\mathcal{T}_{1}+\mathcal{T}_{2},
$$


where

$$
\begin{aligned}
& \mathcal{T}_{1}:=\sup _{t \geq 0} \sup _{x \in[0,1]}\left(\mathrm{e}^{-\beta t} \int_{\mathcal{B}(t)} G_{t-s}(x, y)\left\|b\left(u_{n}(s, y)\right)\right\|_{k} \mathrm{~d} s \mathrm{~d} y\right), \\
& \mathcal{T}_{2}:=\sup _{t \geq 0} \sup _{x \in[0,1]}\left(\mathrm{e}^{-\beta t}\left\|\int_{\mathcal{B}(t)} G_{t-s}(x, y) \sigma\left(u_{n}(s, y)\right) \xi(\mathrm{d} s \mathrm{~d} y)\right\|_{k}\right) .
\end{aligned}
$$

We plan to estimate $\mathcal{T}_{1}$ and $\mathcal{T}_{2}$ in this order.

Recall that $\int_{0}^{1} G_{\rho}(x, y) \mathrm{d} y$ is the probability that Brownian motion, started at $x \in[0,1]$, does not reach the set $\{0,1\}$ before time $\rho>0$. Therefore, the support theorem for the Wiener measure implies that

$$
\int_{0}^{1} G_{\rho}(x, y) \mathrm{d} y<1 \quad \text { for all } \rho>0 \text { and } x \in[0,1] .
$$

From this and (3.1), we can deduce that

$$
\begin{aligned}
\mathcal{T}_{1} & \leq c(b) \sup _{t \geq 0}\left(t \mathrm{e}^{-\beta t}\right)+\mathrm{L}(b) \sup _{t \geq 0} \sup _{x \in[0,1]}\left(\mathrm{e}^{-\beta t} \int_{\mathcal{B}(t)} G_{t-s}(x, y)\left\|u_{n}(s, y)\right\|_{k} \mathrm{~d} s \mathrm{~d} y\right) \\
& =\frac{c(b)}{\mathrm{e} \beta}+\mathrm{L}(b) \sup _{t \geq 0} \sup _{x \in[0,1]}\left(\int_{\mathcal{B}(t)} \mathrm{e}^{-\beta(t-s)} G_{t-s}(x, y) \mathrm{e}^{-\beta s}\left\|u_{n}(s, y)\right\|_{k} \mathrm{~d} s \mathrm{~d} y\right) \\
& \leq \frac{c(b)}{\beta}+\mathrm{L}(b) \mathcal{N}_{\beta, k}\left(u_{n}\right) \cdot \sup _{t \geq 0} \sup _{x \in[0,1]}\left(\int_{\mathcal{B}(t)} \mathrm{e}^{-\beta(t-s)} G_{t-s}(x, y) \mathrm{d} s \mathrm{~d} y\right) .
\end{aligned}
$$

Another appeal to (3.8) yields the following inequality, which is our desired bound for the quantity $\mathcal{T}_{1}$ :

$$
\begin{aligned}
\mathcal{T}_{1} & \leq \frac{c(b)}{\beta}+\mathrm{L}(b) \mathcal{N}_{\beta, k}\left(u_{n}\right) \cdot \sup _{t \geq 0}\left(\int_{0}^{t} \mathrm{e}^{-\beta(t-s)} \mathrm{d} s\right) \\
& \leq \frac{c(b)+\mathrm{L}(b) \mathcal{N}_{\beta, k}\left(u_{n}\right)}{\beta} .
\end{aligned}
$$

In order to estimate $\mathcal{T}_{2}$, we first recall that

$$
\begin{aligned}
& \| \int_{\mathcal{B}(t)} G_{t-s}(x, y) \sigma\left(u_{n}(s, y)\right) \xi(\mathrm{d} s \mathrm{~d} y) \|_{k}^{2} \\
& \quad \leq 4 k \int_{\mathcal{B}(t)}\left[G_{t-s}(x, y)\right]^{2}\left\|\sigma\left(u_{n}(s, y)\right)\right\|_{k}^{2} \mathrm{~d} s \mathrm{~d} y,
\end{aligned}
$$

thanks to a suitable application of the BDG inequality (see [19], Proposition 4.4, p. 36). An appeal to (3.1) yields

$$
\left\|\sigma\left(u_{n}(s, y)\right)\right\|_{k} \leq c(\sigma)+\mathrm{L}(\sigma)\left\|u_{n}(s, y)\right\|_{k} \leq c(\sigma)+\mathrm{L}(\sigma) \mathrm{e}^{\beta s} \mathcal{N}_{\beta, k}\left(u_{n}\right) .
$$


Thus, we see that

$$
\begin{aligned}
\| \int_{\mathcal{B}(t)} & G_{t-s}(x, y) \sigma\left(u_{n}(s, y)\right) \xi(\mathrm{d} s \mathrm{~d} y) \|_{k}^{2} \\
\leq & 4 k \int_{\mathcal{B}(t)}\left[G_{t-s}(x, y)\right]^{2}\left(c(\sigma)+\mathrm{L}(\sigma) \mathrm{e}^{\beta s} \mathcal{N}_{\beta, k}\left(u_{n}\right)\right)^{2} \mathrm{~d} s \mathrm{~d} y \\
\leq & 8 k c(\sigma)^{2} \int_{\mathcal{B}(t)}\left[G_{s}(x, y)\right]^{2} \mathrm{~d} s \mathrm{~d} y \\
& +8 k[\mathrm{~L}(\sigma)]^{2}\left[\mathcal{N}_{\beta, k}\left(u_{n}\right)\right]^{2} \int_{\mathcal{B}(t)} \mathrm{e}^{2 \beta s}\left[G_{t-s}(x, y)\right]^{2} \mathrm{~d} s \mathrm{~d} y .
\end{aligned}
$$

Next, we observe that, uniformly for all $t, \beta>0$,

$$
\int_{\mathcal{B}(t)}\left[G_{S}(x, y)\right]^{2} \mathrm{~d} s \mathrm{~d} y \leq \mathrm{e}^{2 \beta t} \int_{\mathcal{B}(t)} \mathrm{e}^{-2 \beta s}\left[G_{S}(x, y)\right]^{2} \mathrm{~d} s \mathrm{~d} y \lesssim \frac{\mathrm{e}^{2 \beta t}}{\sqrt{\beta}},
$$

where the final bound is justified by Lemma A.2, with the implied universal constant being equal to $(\pi \sqrt{2})^{-1}$. Similarly,

$$
\mathrm{e}^{-2 \beta t} \int_{\mathcal{B}(t)} \mathrm{e}^{2 \beta s}\left[G_{t-s}(x, y)\right]^{2} \mathrm{~d} s \mathrm{~d} y=\int_{\mathcal{B}(t)} \mathrm{e}^{-2 \beta s}\left[G_{s}(x, y)\right]^{2} \mathrm{~d} s \mathrm{~d} y \lesssim \frac{1}{\sqrt{\beta}},
$$

uniformly for all $t, \beta>0$. Consequently,

$$
\begin{aligned}
\mathrm{e}^{-2 \beta t} & \left\|\int_{\mathcal{B}(t)} G_{t-s}(x, y) \sigma\left(u_{n}(s, y)\right) \xi(\mathrm{d} s \mathrm{~d} y)\right\|_{k}^{2} \\
& \lesssim \frac{k c(\sigma)^{2}+k[\mathrm{~L}(\sigma)]^{2}\left[\mathcal{N}_{\beta, k}\left(u_{n}\right)\right]^{2}}{\sqrt{\beta}}
\end{aligned}
$$

uniformly for all $n \geq 0, x \in[0,1], \beta>0$ and $k \geq 2$. We take square roots of both sides, then optimize over $t \geq 0$ and $x \in[0,1]$ in order to see that

$$
\mathcal{T}_{2} \lesssim \frac{k^{1 / 2}}{\beta^{1 / 4}} \cdot\left(c(\sigma)+\mathrm{L}(\sigma) \mathcal{N}_{\beta, k}\left(u_{n}\right)\right)
$$

with the same uniformity properties as before on $(k, \beta, x, n)$. This is the desired inequality for $\mathcal{T}_{2}$.

We now combine (3.7) with (3.9) and (3.10) in order to see that

$$
\mathcal{N}_{\beta, k}\left(u_{n+1}\right) \leq K_{\beta, k}+L_{\beta, k} \mathcal{N}_{\beta, k}\left(u_{n}\right),
$$

uniformly for all $\beta>0, k \geq 2$, and $n \geq 0$, where

$$
\begin{aligned}
K_{\beta, k} & :=c\left(\left\|u_{0}\right\|_{\mathbb{L}^{\infty}}+\frac{c(b)}{\beta}+\frac{k^{1 / 2} c(\sigma)}{\beta^{1 / 4}}\right), \\
L_{\beta, k} & :=c \max \left(\frac{\mathrm{L}(b)}{\beta}, \frac{k^{1 / 2} \mathrm{~L}(\sigma)}{\beta^{1 / 4}}\right),
\end{aligned}
$$


for a sufficiently-large finite universal constant $c>1$. Let us choose $\beta:=16 c^{4} \mathrm{~L}(b)$ and observe that, for this choice of $\beta$,

$$
\begin{aligned}
K_{16 c^{4} \mathrm{~L}(b), k} & \leq c\left\|u_{0}\right\|_{\mathbb{L}^{\infty}}+\frac{c(b)}{16 \mathrm{~L}(b)}+\frac{k^{1 / 2} c(\sigma)}{2[\mathrm{~L}(b)]^{1 / 4}}, \\
L_{16 c^{4} \mathrm{~L}(b), k} & \leq \max \left(\frac{1}{16}, \frac{k^{1 / 2} \mathrm{~L}(\sigma)}{2[\mathrm{~L}(b)]^{1 / 4}}\right) .
\end{aligned}
$$

In this way, we may simplify (3.11) to the following recursive inequality: Uniformly for all integers $n \geq 0$ and real numbers $k \in\left[2, \sqrt{\mathrm{L}(b)} / \mathrm{L}(\sigma)^{2}\right]$,

$$
\mathcal{N}_{16 c^{4} \mathrm{~L}(b), k}\left(u_{n+1}\right) \leq c\left\|u_{0}\right\|_{\mathbb{L}^{\infty}}+\frac{c(b)}{16 \mathrm{~L}(b)}+\frac{c(\sigma)}{2 \mathrm{~L}(\sigma)}+\frac{1}{2} \mathcal{N}_{16 c^{4} \mathrm{~L}(b), k}\left(u_{n}\right) .
$$

Since $\mathcal{N}_{16 c^{4} \mathrm{~L}(b), k}\left(u_{0}\right)=\left\|u_{0}\right\|_{\mathbb{L}^{\infty}}$ is finite, the preceding implies that $\sup _{n \geq 0} \mathcal{N}_{\beta, k}\left(u_{n}\right)<\infty$ for all real numbers $k \in\left[2, \sqrt{\mathrm{L}(b)} / \mathrm{L}(\sigma)^{2}\right]$, and, more significantly,

$$
\limsup _{n \rightarrow \infty} \mathcal{N}_{16 c^{4} \mathrm{~L}(b), k}\left(u_{n}\right) \leq 2 c\left\|u_{0}\right\|_{\mathbb{L}^{\infty}}+\frac{c(b)}{8 \mathrm{~L}(b)}+\frac{c(\sigma)}{\mathrm{L}(\sigma)} .
$$

By Fatou's lemma and (3.5),

$$
\begin{aligned}
\mathcal{N}_{16 c^{4} \mathrm{~L}(b), k}(u) & \leq \limsup _{n \rightarrow \infty} \mathcal{N}_{16 c^{4} \mathrm{~L}(b), k}\left(u_{n}\right) \\
& \lesssim\left\|u_{0}\right\|_{\mathbb{L}^{\infty}}+\frac{c(b)}{\mathrm{L}(b)}+\frac{c(\sigma)}{\mathrm{L}(\sigma)},
\end{aligned}
$$

uniformly for all $k \in\left[2, \sqrt{\mathrm{L}(b)} / \mathrm{L}(\sigma)^{2}\right]$. We can unscramble this inequality directly, using only (3.6), in order to deduce the proposition.

In the context of Proposition 3.1, one might wonder about the moments of order $k$ when $k>\sqrt{\mathrm{L}(b)} / \mathrm{L}(\sigma)^{2}$. In that case, it is possible to adjust only slightly the proof of Proposition 3.1 in order to obtain the following improvement of [19], Theorem 5.5.

PROPOSITION 3.3. If $L(b) \geq 4 L(\sigma)^{4}>0$, then there exists a finite universal constant A such that

$$
\sup _{x \in[0,1]} \mathrm{E}\left(|u(t, x)|^{k}\right) \leq A^{k}\left(\left\|u_{0}\right\|_{\mathbb{L}^{\infty}}+\frac{c(b)}{[L(\sigma)]^{4}}+\frac{c(\sigma)}{L(\sigma)}\right)^{k} \cdot \exp \left(A k^{3}[L(\sigma)]^{4} t\right),
$$

uniformly for all real numbers $t \geq 0$ and $k>\sqrt{L(b)} / L(\sigma)^{2}$.

Proof. We follow the proof of Proposition 3.1 up to and including (3.11) without change. However, if $k>\sqrt{\mathrm{L}(b)} / \mathrm{L}(\sigma)^{2}$, then we choose the auxiliary parameter $\beta$ slightly differently. Namely, let us define

$$
\beta:=16 c^{4} k^{2}[\mathrm{~L}(\sigma)]^{4},
$$


notation being that of (3.11). For this particular choice,

$$
K_{\beta, k}=c\left\|u_{0}\right\|_{\mathbb{L}^{\infty}}+\frac{c(b)}{16 c^{3} k^{2}[\mathrm{~L}(\sigma)]^{4}}+\frac{c(\sigma)}{2 \mathrm{~L}(\sigma)},
$$

and $L_{\beta, k}=\frac{1}{2}$. We apply the preceding particular choice of $\beta$ in (3.11) in order to see that

$$
\mathcal{N}_{\beta, k}\left(u_{n+1}\right) \leq K_{\beta, k}+\frac{1}{2} \mathcal{N}_{\beta, k}\left(u_{n}\right),
$$

for all $n \geq 0$. This shows in particular that $\mathcal{N}_{\beta, k}\left(u_{n}\right) \lesssim K_{\beta, k}$ uniformly for all $n \geq 0$, which is another way to state the result.

3.2. An optimal regularity theorem. Next, we derive the following optimal regularity result. This improves the classical Hölder-continuity statement of [28], Corollary 3.4 (see also [10], (2.3)). A related statement is given in [6], Theorem 3.1, for the stochastic heat equation on the whole real line.

THEOREM 3.4. The following logical implications are valid:

$$
\alpha \in\left(0, \frac{1}{2}\right), u_{0} \in \mathbb{C}_{0}^{\alpha} \quad \Longrightarrow \quad \mathrm{P}\left\{u(t) \in \mathbb{C}_{0}^{\alpha} \text { for all } t>0\right\}=1
$$

and

$$
\alpha \in\left[\frac{1}{2}, 1\right], u_{0} \in \mathbb{C}_{0}^{\alpha} \Longrightarrow \mathrm{P}\left\{u(t) \in \bigcap_{\varepsilon>0} \mathbb{C}_{0}^{\frac{1}{2}-\varepsilon} \text { for all } t>0\right\}=1
$$

We begin by establishing some quantitive estimates that describe the smoothness properties of the solution to (1.1). Clearly, this work prepares for Theorem 3.4, since among other things, Theorem 3.4 asserts that the solution to (1.1) is Hölder continuous.

Let us first observe that (2.4) identifies every kernel $G_{t}$ with a linear operator $\mathcal{G}_{t}$ in the usual way. It is well known - and easy to verify directly using (2.4) — that $\left\{\mathcal{G}_{t}\right\}_{t \geq 0}$ is a semigroup of linear operators that is bounded in $\mathbb{L}^{\infty}$ and $\left(\mathcal{G}_{t} \mathbb{1}\right)(x) \leq 1$ for all $x \in[0,1]$ and $t \geq 0$, where $\mathbb{1}(x):=x$ for all $x \in[0,1]$.

The semigroup $\left\{\mathcal{G}_{t}\right\}_{t \geq 0}$ is said to be Feller uniformly on a function class $\mathcal{F}$ if

$$
\lim _{t \downarrow 0} \sup _{f \in \mathcal{F}} \sup _{x \in[0,1]}\left|\left(\mathcal{G}_{t} f\right)(x)-f(x)\right|=0 .
$$

The following lemma shows that $\left\{\mathcal{G}_{t}\right\}_{t \geq 0}$ is indeed Feller uniformly on every bounded subset $\mathcal{F}$ of $\mathbb{C}_{0}^{\alpha}$ for every $\alpha \in(0,1]$. In fact, the following contains the quantitative improvement,

$$
\sup _{f \in \mathcal{F}} \sup _{x \in[0,1]}\left|\left(\mathcal{G}_{t} f\right)(x)-f(x)\right|=O\left(t^{\alpha / 2}\right) \quad \text { as } t \downarrow 0,
$$

for every bounded subset $\mathcal{F}$ of the Banach space $\mathbb{C}_{0}^{\alpha}$. 
LEMmA 3.5 (A quantitative Feller property). Choose and fix $\alpha \in(0,1]$. Then

$$
\sup _{x \in[0,1]} \sup _{t \geq 0} \frac{\left|\left(\mathcal{G}_{t} f\right)(x)-f(x)\right|}{t^{\alpha / 2}} \lesssim\|f\|_{\mathbb{C}_{0}^{\alpha}},
$$

uniformly for all $f \in \mathbb{C}_{0}^{\alpha}$.

PROOF. Let us choose and fix $t>0$ and $x \in[0,1]$. Then

$$
\begin{aligned}
\mid\left(\mathcal{G}_{t} f\right) & (x)-f(x) \mid \\
= & \left|\int_{0}^{1} G_{t}(x, z) f(z) \mathrm{d} z-f(x)\right| \\
= & \left|\int_{0}^{1} G_{t}(x, z)[f(z)-f(x)] \mathrm{d} z+f(x)\left(\int_{0}^{1} G_{t}(x, z) \mathrm{d} z-1\right)\right| \\
& \leq \int_{0}^{1} G_{t}(x, z)\|f\|_{\mathbb{C}_{0}^{\alpha}}|z-x|^{\alpha} \mathrm{d} z+|f(x)|\left[1-\int_{0}^{1} G_{t}(x, z) \mathrm{d} z\right] .
\end{aligned}
$$

Use the inequality (2.6) for the first term, and the fact that

$$
|f(x)|=|f(x)-f(0)|=|f(x)-f(1)| \leq\|f\|_{\mathbb{C}_{0}^{\alpha}}(\min (x, 1-x))^{\alpha},
$$

for the second term, in order to see that

$$
\left|\left(\mathcal{G}_{t} f\right)(x)-f(x)\right| \leq c_{0}\|f\|_{\mathbb{C}_{0}^{\alpha}} t^{\alpha / 2}+\|f\|_{\mathbb{C}_{0}^{\alpha}}(\min (x, 1-x))^{\alpha}\left(1-\int_{0}^{1} G_{t}(x, z) \mathrm{d} z\right),
$$

where $c_{0}$ is a universal constant.

Let $B:=\left\{B_{t}\right\}_{t \geq 0}$ denote a standard 1-dimensional Brownian motion, and consider the (a.s. finite) stopping time

$$
\tau:=\inf \left\{t>0: B_{t} \in\{0,1\}\right\} .
$$

Thus, we may write, using standard notation: For all $x \in[0,1]$ and $t>0$,

$$
\begin{aligned}
1-\int_{0}^{1} G_{t}(x, z) \mathrm{d} z & =\mathrm{P}_{x}\{\tau \leq t\}=\mathrm{P}_{0}\left\{\sup _{s \in[0,1]}\left|B_{s}\right| \geq \frac{\min (x, 1-x)}{t^{1 / 2}}\right\} \\
& \leq \exp \left[-\frac{(\min (x, 1-x))^{2}}{2 t}\right] .
\end{aligned}
$$

Let $y:=\min (x, 1-x)$. By the last inequality,

$$
\begin{aligned}
y^{\alpha}\left(1-\int_{0}^{1} G_{t}(x, z) \mathrm{d} z\right) & \leq y^{\alpha} \exp \left[-\frac{y^{2}}{2 t}\right] \\
& \leq t^{\alpha / 2}\left(\frac{y^{2}}{t}\right)^{\alpha / 2} \exp \left[-\frac{y^{2}}{2 t}\right] \leq c t^{\alpha / 2} .
\end{aligned}
$$


We conclude that

$$
\left|\left(\mathcal{G}_{t} f\right)(x)-f(x)\right| \leq \tilde{c}_{\alpha}\|f\|_{\mathbb{C}_{0}^{\alpha}} t^{\alpha / 2},
$$

where $\tilde{c}_{\alpha}=1+\sup _{y \geq 0}\left(y^{\alpha / 2} \mathrm{e}^{-y / 2}\right)=1+(\alpha / \mathrm{e})^{\alpha / 2}$.

REMARK 3.6. Suppose that $f(y)=\sum_{n=1}^{\infty} f_{n} \sin (n \pi y)$ for $y \in[0,1]$, where the Fourier sine coefficients $\left\{f_{n}\right\}_{n=1}^{\infty}$ of $f$ satisfy $\|f\|_{1, \alpha}:=\sum_{n=1}^{\infty}\left|f_{n}\right| n^{\alpha}<\infty$ for some $\alpha \in(0,1]$. Then it is not hard to see that $f \in \mathbb{C}_{0}^{\alpha}$ and $\|f\|_{\mathbb{C}_{0}^{\alpha}} \leq \pi^{\alpha}\|f\|_{1, \alpha}$. Indeed, $f$ vanishes on $\{0,1\}$, and for every distinct $x, y \in[0,1]$,

$$
\frac{|f(x)-f(y)|}{|x-y|^{\alpha}} \leq \sum_{n=1}^{\infty}\left|f_{n}\right| \cdot \frac{|\sin (n \pi x)-\sin (n \pi y)|}{|x-y|^{\alpha}} \leq \pi^{\alpha}\|f\|_{1, \alpha} .
$$

In this particular case, a simpler argument than the proof of Lemma 3.5 yields the slightly weaker bound,

$$
\sup _{t>0} \sup _{x \in[0,1]} \frac{\left|\left(\mathcal{G}_{t} f\right)(x)-f(x)\right|}{t^{\alpha / 2}} \lesssim\|f\|_{1, \alpha} .
$$

Indeed, $\left(\mathcal{G}_{t} f\right)(x)=\int_{0}^{1} G_{t}(x, y) f(y) \mathrm{d} y=\sum_{n=1}^{\infty} f_{n} \sin (n \pi x) \exp \left(-n^{2} \pi^{2} t / 2\right)$, and so

$$
\begin{aligned}
\left|\left(\mathcal{G}_{t} f\right)(x)-f(x)\right| & \leq\left|\sum_{n=1}^{\infty} f_{n} \sin (n \pi x)\left(1-\exp \left(-n^{2} \pi^{2} t / 2\right)\right)\right| \\
& \leq \frac{\pi^{\alpha} t^{\alpha / 2}}{2^{\alpha / 2}} \sum_{n=1}^{\infty}\left|f_{n}\right| n^{\alpha}\left|\frac{1-\exp \left(-n^{2} \pi^{2} t / 2\right)}{\left(n^{2} \pi^{2} t / 2\right)^{\alpha / 2}}\right| \\
& \leq \frac{C_{\alpha} \pi^{\alpha}}{2^{\alpha / 2}} \cdot\|f\|_{1, \alpha} t^{\alpha / 2}
\end{aligned}
$$

where $C_{\alpha}=\sup _{y>0} y^{-\alpha / 2}(1-\exp (-y))<\infty$.

The next result is also a deterministic lemma. Among other things, it asserts that every $\mathcal{G}_{t}$ maps each $\mathbb{C}_{0}^{\alpha}$ boundedly to $\mathbb{C}_{0}^{\alpha}$.

LEMMA 3.7. Choose and fix an arbitrary $\alpha \in(0,1]$. Then

$$
\sup _{0 \leq x<x^{\prime} \leq 1} \sup _{t \geq 0} \frac{\left|\left(\mathcal{G}_{t} f\right)(x)-\left(\mathcal{G}_{t} f\right)\left(x^{\prime}\right)\right|}{\left|x-x^{\prime}\right|^{\alpha}} \lesssim\|f\|_{\mathbb{C}_{0}^{\alpha}},
$$

uniformly for all $f \in \mathbb{C}_{0}^{\alpha}$.

PROOF. It is well known that the Green's function $G$ can be represented as follows:

$$
G_{t}(x, z)=\varphi_{t}(z-x)-\varphi_{t}(z+x) \quad \text { for all } t>0 \text { and } x, z \in(0,1),
$$


where

$$
\varphi_{t}(x):=\frac{1}{\sqrt{2 \pi t}} \sum_{n=-\infty}^{\infty} \exp \left[-\frac{(x-n)^{2}}{2 t}\right]
$$

see, for example, Bally et al. [1], Proof of Lemma A2.

Choose and fix $x, y \in[0,1]$ such that

$$
h:=y-x>0 .
$$

Thanks to (3.14),

$$
\begin{aligned}
\left(\mathcal{G}_{t} f\right)(x)-\left(\mathcal{G}_{t} f\right)(y) & \\
= & \int_{0}^{1}\left[G_{t}(x, z)-G_{t}(y, z)\right] f(z) \mathrm{d} z \\
= & \int_{0}^{1}\left[\varphi_{t}(z-x)-\varphi_{t}(z-h-x)\right] f(z) \mathrm{d} z \\
& \quad-\int_{0}^{1}\left[\varphi_{t}(z+x)-\varphi_{t}(z+h+x)\right] f(z) \mathrm{d} z
\end{aligned}
$$

for all $t>0$. We follow Bally et al. [1] and organize the preceding as follows:

$$
\left(\mathcal{G}_{t} f\right)(x)-\left(\mathcal{G}_{t} f\right)(y)=\mathcal{I}_{1}-\mathcal{I}_{2}+\mathcal{I}_{3}-\mathcal{I}_{4}-\mathcal{I}_{5}+\mathcal{I}_{6},
$$

where

$$
\begin{aligned}
& \mathcal{I}_{1}=\int_{0}^{1-h} \varphi_{t}(z-x)[f(z)-f(z+h)] \mathrm{d} z \\
& \mathcal{I}_{2}=\int_{h}^{1} \varphi_{t}(z+x)[f(z)-f(z-h)] \mathrm{d} z \\
& \mathcal{I}_{3}=\int_{1-h}^{1} \varphi_{t}(z-x) f(z) \mathrm{d} z \\
& \mathcal{I}_{4}=\int_{-h}^{0} \varphi_{t}(z-x) f(z+h) \mathrm{d} z \\
& \mathcal{I}_{5}=\int_{0}^{h} \varphi_{t}(z+x) f(z) \mathrm{d} z \\
& \mathcal{I}_{6}=\int_{1}^{1+h} \varphi_{t}(z+x) f(z-h) \mathrm{d} z .
\end{aligned}
$$

Since $f \in \mathbb{C}_{0}^{\alpha}$ and $\varphi_{t}$ is a probability density, $\left|\mathcal{I}_{\ell}\right| \leq h^{\alpha}\|f\|_{\mathbb{C}_{0}^{\alpha}}$ for $\ell=1,2$. Moreover, we can replace $f(z)$ by $f(z)-f(1)$ in $\mathcal{I}_{3}$ and $\mathcal{I}_{6}$, and by $f(z)-f(0)$ in $\mathcal{I}_{4}$ 
and $\mathcal{I}_{5}$, in order to obtain

$$
\begin{aligned}
\left|\left(\mathcal{G}_{t} f\right)(x)-\left(\mathcal{G}_{t} f\right)(y)\right| \\
\leq 2 h^{\alpha}\|f\|_{\mathbb{C}_{0}^{\alpha}}+\|f\|_{\mathbb{C}_{0}^{\alpha}}\left[\int_{1-h}^{1} \varphi_{t}(z-x)|z-1|^{\alpha} \mathrm{d} z\right. \\
\quad+\int_{-h}^{0} \varphi_{t}(z-x)|z+h|^{\alpha} \mathrm{d} z \\
\left.\quad+\int_{0}^{h} \varphi_{t}(z+x)|z|^{\alpha} \mathrm{d} z+\int_{1}^{1+h} \varphi_{t}(z+x)|z-h-1|^{\alpha} \mathrm{d} z\right] .
\end{aligned}
$$

Because the absolute values are all bounded above by $h$; the preceding quantity is at most $6 h^{\alpha}\|f\|_{\mathbb{C}_{0}^{\alpha}}$. This completes the proof.

We now begin to use the preceding two analytic results about the Dirichlet Laplacian, acting on $\mathbb{C}_{0}^{\alpha}$, in order to derive smoothness results for the solution to (1.1). First, let us present a result about smoothness in the space variable.

Throughout, we write

$$
u(t, x)=\left(\mathcal{G}_{t} u_{0}\right)(x)+I(t, x),
$$

where

$$
I(t, x)=\int_{\mathcal{B}(t)} G_{t-s}(x, y) b(u(s, y)) \mathrm{d} s \mathrm{~d} y+\int_{\mathcal{B}(t)} G_{t-s}(x, y) \sigma(u(s, y)) \xi(\mathrm{d} s \mathrm{~d} y),
$$

and $\mathcal{B}(t)$ was defined in (3.3). It might help to recall that (3.2) is in place throughout the section.

Proposition 3.8. Choose and fix $\alpha \in(0,1]$. There exists a finite universal constant $A$-independent of $(b, \sigma)$-such that

$$
\sup _{0 \leq x<x^{\prime} \leq 1} \mathrm{E}\left(\left|\frac{I(t, x)-I\left(t, x^{\prime}\right)}{\left|x^{\prime}-x\right|^{1 / 2}}\right|^{k}\right) \leq A^{k}\left(k^{k / 2} \mathcal{M}_{1}^{k}+k^{k / 2} \mathcal{M}_{2}^{k} \mathcal{M}_{3}^{k} \mathrm{e}^{A k L(b) t}\right),
$$

and

$$
\begin{aligned}
& \sup _{0 \leq x<x^{\prime} \leq 1} \mathrm{E}\left(\left|\frac{u(t, x)-u\left(t, x^{\prime}\right)}{\left|x^{\prime}-x\right|^{\alpha \wedge(1 / 2)}}\right|^{k}\right) \\
& \quad \leq A^{k}\left(\left\|u_{0}\right\|_{\mathbb{C}_{0}^{\alpha}}^{k}+k^{k / 2} \mathcal{M}_{1}^{k}+k^{k / 2} \mathcal{M}_{2}^{k} \mathcal{M}_{3}^{k} \mathrm{e}^{A k L(b) t}\right)
\end{aligned}
$$

uniformly for all $u_{0} \in \mathbb{C}_{0}^{\alpha}, t \geq 0$, and $k \in\left[2, \sqrt{L(b)} / L(\sigma)^{2}\right]$, where

$$
\begin{aligned}
& \mathcal{M}_{1}:=c(b)+c(\sigma), \\
& \mathcal{M}_{2}:=L(b)+L(\sigma)
\end{aligned}
$$

and

$$
\mathcal{M}_{3}:=\left\|u_{0}\right\|_{\mathbb{L}^{\infty}}+\frac{c(b)}{L(b)}+\frac{c(\sigma)}{L(\sigma)}
$$


REMARK 3.9. Stated in other words, the above asserts that the moments of order $\leq \sqrt{\mathrm{L}(b)} / \mathrm{L}(\sigma)^{2}$ behave as those of a Gaussian random variable. Of course, such a statement can have nontrivial content only when $\mathrm{L}(b) \gg 4[\mathrm{~L}(\sigma)]^{4}$.

PROOF. Thanks to (3.4), we may write

$$
\left\|u(t, x)-u\left(t, x^{\prime}\right)\right\|_{k} \leq\left|\left(\mathcal{G}_{t} u_{0}\right)(x)-\left(\mathcal{G}_{t} u_{0}\right)\left(x^{\prime}\right)\right|+\left\|I(t, x)-I\left(t, x^{\prime}\right)\right\|_{k},
$$

and

$$
\left\|I(t, x)-I\left(t, x^{\prime}\right)\right\|_{k} \leq\left\|\mathcal{T}_{1}\right\|_{k}+\left\|\mathcal{T}_{2}\right\|_{k}
$$

where

$$
\begin{aligned}
& \mathcal{T}_{1}:=\int_{\mathcal{B}(t)}\left[G_{t-s}(x, y)-G_{t-s}\left(x^{\prime}, y\right)\right] b(u(s, y)) \mathrm{d} s \mathrm{~d} y, \\
& \mathcal{T}_{2}:=\int_{\mathcal{B}(t)}\left[G_{t-s}(x, y)-G_{t-s}\left(x^{\prime}, y\right)\right] \sigma(u(s, y)) \xi(\mathrm{d} s \mathrm{~d} y) .
\end{aligned}
$$

Lemma 3.7 estimates the first term on the right-hand side of (3.15) as follows:

$$
\left|\left(\mathcal{G}_{t} u_{0}\right)(x)-\left(\mathcal{G}_{t} u_{0}\right)\left(x^{\prime}\right)\right| \lesssim\left\|u_{0}\right\|_{\mathbb{C}_{0}^{\alpha}} \cdot\left|x-x^{\prime}\right|^{\alpha},
$$

uniformly for all $t \geq 0$ and $x, x^{\prime} \in[0,1]$.

Next, we estimate $\mathcal{T}_{1}$. First, an appeal to (3.1) yields

$$
\left\|\mathcal{T}_{1}\right\|_{k} \leq \int_{\mathcal{B}(t)}\left|G_{t-s}(x, y)-G_{t-s}\left(x^{\prime}, y\right)\right|\left(c(b)+\mathrm{L}(b)\|u(s, y)\|_{k}\right) \mathrm{d} s \mathrm{~d} y .
$$

Lemma A. 3 ensures that, for all $x, x^{\prime} \in[0,1]$,

$$
\sup _{t>0} \int_{\mathcal{B}(t)}\left|G_{s}(x, y)-G_{s}\left(x^{\prime}, y\right)\right| \mathrm{d} s \mathrm{~d} y \lesssim\left|x-x^{\prime}\right| \log _{+}\left(\frac{1}{\left|x-x^{\prime}\right|}\right),
$$

where $\log _{+}(a):=\log (\mathrm{e} \vee a)$ for all $a \in \mathbb{R}$. Furthermore,

$$
\begin{aligned}
\int_{\mathcal{B}(t)} & \left|G_{t-s}(x, y)-G_{t-s}\left(x^{\prime}, y\right)\right| \cdot\|u(s, y)\|_{k} \mathrm{~d} s \mathrm{~d} y \\
& \leq \mathrm{e}^{\beta t} \mathcal{N}_{\beta, k}(u) \sup _{t>0} \int_{\mathcal{B}(t)}\left|G_{t-s}(x, y)-G_{t-s}\left(x^{\prime}, y\right)\right| \mathrm{d} s \mathrm{~d} y \\
& \lesssim \mathrm{e}^{\beta t} \mathcal{N}_{\beta, k}(u) \cdot\left|x-x^{\prime}\right| \log _{+}\left(\frac{1}{\left|x-x^{\prime}\right|}\right),
\end{aligned}
$$

thanks to a second appeal to Lemma A.3. [The norm $\mathcal{N}_{\beta, k}$ was defined in (3.6).] It follows that

$$
\begin{aligned}
\left\|\mathcal{T}_{1}\right\|_{k} & \leq\left[c(b)+\mathrm{L}(b) \mathrm{e}^{\beta t} \mathcal{N}_{\beta, k}(u)\right] \cdot\left|x-x^{\prime}\right| \log _{+}\left(\frac{1}{\left|x-x^{\prime}\right|}\right) \\
& \lesssim\left[c(b)+\mathrm{L}(b) \mathrm{e}^{\beta t} \mathcal{N}_{\beta, k}(u)\right] \cdot\left|x-x^{\prime}\right|^{1 / 2}
\end{aligned}
$$


The last line follows from the elementary fact that $|a| \log _{+}(a) \lesssim|a|^{1 / 2}$ for all $a \in$ $[-1,1]$, and the above inequality yields the desired bound for the $L^{k}(\Omega)$-norm of $\mathcal{T}_{1}$.

For the corresponding estimate for $\mathcal{T}_{2}$, use the BDG inequality as follows:

$$
\left\|\mathcal{T}_{2}\right\|_{k}^{2} \leq 4 k \int_{\mathcal{B}(t)}\left|G_{t-s}(x, y)-G_{t-s}\left(x^{\prime}, y\right)\right|^{2}\left(c(\sigma)+\mathrm{L}(\sigma)\|u(s, y)\|_{k}\right)^{2} \mathrm{~d} s \mathrm{~d} y
$$

see the proof of Proposition 3.1 for more details on the justification of this sort of inequality. Now, Lemma A.3 below tells us that

$$
\sup _{t>0} \int_{\mathcal{B}(t)}\left|G_{s}(x, y)-G_{s}\left(x^{\prime}, y\right)\right|^{2} \mathrm{~d} s \mathrm{~d} y \lesssim\left|x-x^{\prime}\right| .
$$

Also,

$$
\begin{aligned}
\int_{\mathcal{B}(t)} & \left|G_{t-s}(x, y)-G_{t-s}\left(x^{\prime}, y\right)\right|^{2}\|u(s, y)\|_{k}^{2} \mathrm{~d} s \mathrm{~d} y \\
& \leq \mathrm{e}^{2 \beta t}\left[\mathcal{N}_{\beta, k}(u)\right]^{2} \sup _{t>0} \int_{\mathcal{B}(t)}\left|G_{s}(x, y)-G_{s}\left(x^{\prime}, y\right)\right|^{2} \mathrm{~d} s \mathrm{~d} y \\
& \lesssim \mathrm{e}^{2 \beta t}\left[\mathcal{N}_{\beta, k}(u)\right]^{2} \cdot\left|x-x^{\prime}\right| .
\end{aligned}
$$

Therefore, it follows from the preceding development that

$$
\left\|\mathcal{T}_{2}\right\|_{k} \lesssim \sqrt{k}\left[c(\sigma)+\mathrm{L}(\sigma) \mathrm{e}^{\beta t} \mathcal{N}_{\beta, k}(u)\right] \cdot\left|x-x^{\prime}\right|^{1 / 2}
$$

This is the desired estimate of $\mathcal{T}_{2}$.

We can now combine (3.16), (3.18) and (3.19) in order to see that

$$
\begin{aligned}
\frac{\left\|I(t, x)-I\left(t, x^{\prime}\right)\right\|_{k}}{\left|x-x^{\prime}\right|^{1 / 2}} & \leq[c(b)+\sqrt{k} c(\sigma)]+\mathrm{e}^{\beta t}[\mathrm{~L}(b)+\sqrt{k} \mathrm{~L}(\sigma)] \mathcal{N}_{\beta, k}(u) \\
& \leq \sqrt{k} \mathcal{M}_{1}+\sqrt{k} \mathrm{e}^{\beta t} \mathcal{M}_{2} \mathcal{N}_{\beta, k}(u) .
\end{aligned}
$$

Together with (3.15) and (3.17),

$$
\begin{aligned}
& \frac{\left\|u(t, x)-u\left(t, x^{\prime}\right)\right\|_{k}}{\mid x}-\left.x^{\prime}\right|^{\alpha \wedge(1 / 2)} \\
& \quad \lesssim\left\|u_{0}\right\|_{\mathbb{C}_{0}^{\alpha}}+[c(b)+\sqrt{k} c(\sigma)]+\mathrm{e}^{\beta t}[\mathrm{~L}(b)+\sqrt{k} \mathrm{~L}(\sigma)] \mathcal{N}_{\beta, k}(u) \\
& \quad \leq\left\|u_{0}\right\|_{\mathbb{C}_{0}^{\alpha}}+\sqrt{k} \mathcal{M}_{1}+\sqrt{k} \mathrm{e}^{\beta t} \mathcal{M}_{2} \mathcal{N}_{\beta, k}(u),
\end{aligned}
$$

uniformly for all $t \geq 0, \beta>0$, distinct $x, x^{\prime} \in[0,1]$, and $k \geq 2$. We apply the preceding with the particular choice,

$$
\beta:=16 c^{4} \mathrm{~L}(b),
$$


where $c \in(0, \infty)$ is the same universal constant that arose in (3.12). Proposition 3.1 [see in particular the equivalent formulation (3.12)] now tells us that

$$
\frac{\left\|I(t, x)-I\left(t, x^{\prime}\right)\right\|_{k}}{\left|x-x^{\prime}\right|^{1 / 2}} \lesssim \sqrt{k} \mathcal{M}_{1}+\sqrt{k} \mathrm{e}^{16 c^{4} \mathrm{~L}(b) t} \mathcal{M}_{2} \mathcal{M}_{3},
$$

and

$$
\frac{\left\|u(t, x)-u\left(t, x^{\prime}\right)\right\|_{k}}{\left|x-x^{\prime}\right|^{\alpha \wedge(1 / 2)}} \lesssim\left\|u_{0}\right\|_{\mathbb{C}_{0}^{\alpha}}+\sqrt{k} \mathcal{M}_{1}+\sqrt{k} \mathrm{e}^{16 c^{4} \mathrm{~L}(b) t} \mathcal{M}_{2} \mathcal{M}_{3},
$$

uniformly for all $t \geq 0$, distinct $x, x^{\prime} \in[0,1]$, and $k \in\left[2, \sqrt{\mathrm{L}(b)} / \mathrm{L}(\sigma)^{2}\right]$. This is equivalent to the statement of the proposition.

Proposition 3.8 has a counterpart when $k>\sqrt{\mathrm{L}(b)} / \mathrm{L}(\sigma)^{2}$. We will need only the following crude version of such a counterpart.

Proposition 3.10. If $u_{0} \in \mathbb{C}_{0}^{\alpha}$ for some $\alpha \in(0,1]$, then

$$
\sup _{t \geq 0} \sup _{0 \leq x<x^{\prime} \leq 1} \mathrm{E}\left(\left|\frac{u(t, x)-u\left(t, x^{\prime}\right)}{\left(x^{\prime}-x\right)^{\alpha \wedge(1 / 2)}}\right|^{k}\right)<\infty \quad \text { for all } k \geq 2 .
$$

PROOF. We merely adjust the proof of Proposition 3.8 by using in (3.20) the result of Proposition 3.3, instead of Proposition 3.1, in order to bound $\mathcal{N}_{\beta, k}(u)$. More concretely, we use the same argument that we used to prove Proposition 3.8, but with $\beta=16 c^{4} k^{2}[\mathrm{~L}(\sigma)]^{4}$ instead of $\beta=16 c^{4} \mathrm{~L}(b)$ in that proof. Then we follow through the remainder of the derivation, making only small arithmetic adjustments for the new choice of $\beta$.

Next, we derive an a priori smoothness estimate for the temporal behavior of the solution to (1.1).

Proposition 3.11. Fix $T_{0}>0$. Choose and fix some $\alpha \in(0,1]$, and define $\mu:=\min \left(\frac{1}{4}, \frac{1}{2} \alpha\right)$. Then there exists a finite constant $A$-independent of $(b, \sigma)$ such that

$$
\sup _{x \in[0,1]} \mathrm{E}\left(\left|\frac{u(T, x)-u(t, x)}{(T-t)^{\mu}}\right|^{k}\right) \leq A^{k}\left(\left\|u_{0}\right\|_{\mathbb{C}_{0}^{\alpha}}^{k}+k^{k / 2}\left[\mathcal{M}_{1}^{k}+\mathcal{M}_{2}^{k} \mathcal{M}_{3}^{k} \mathrm{e}^{A k L(b)(T)}\right]\right),
$$

for all $u_{0} \in \mathbb{C}_{0}^{\alpha}, 0 \leq t<T \leq T_{0}$, and $k \in\left[2, \sqrt{L(b)} / L(\sigma)^{2}\right]$.

One can make a remark, similar to Remark 3.9, about the Gaussian nature of the large moments of the temporal increments of $u$ in the case that $\mathrm{L}(b) \gg[\mathrm{L}(\sigma)]^{4}$.

Proof. Let $T>t>0$ and $x \in[0,1]$ be fixed; the case $t=0$ is similar but simpler. In a manner similar to (3.15), we have

$\|u(T, x)-u(t, x)\|_{k} \leq\left|\left(\mathcal{G}_{T} u_{0}\right)(x)-\left(\mathcal{G}_{t} u_{0}\right)(x)\right|+\left\|\mathcal{T}_{1}\right\|_{k}+\left\|\mathcal{T}_{2}\right\|_{k}+\left\|\mathcal{T}_{3}\right\|_{k}+\left\|\mathcal{T}_{4}\right\|_{k}$, 
where

$$
\begin{aligned}
& \mathcal{T}_{1}:=\int_{(0, t) \times(0,1)}\left[G_{T-s}(x, y)-G_{t-s}(x, y)\right] b(u(s, y)) \mathrm{d} s \mathrm{~d} y, \\
& \mathcal{T}_{2}:=\int_{(t, T) \times[0,1]} G_{T-s}(x, y) b(u(s, y)) \mathrm{d} s \mathrm{~d} y, \\
& \mathcal{T}_{3}:=\int_{(0, t) \times(0,1)}\left[G_{T-s}(x, y)-G_{t-s}(x, y)\right] \sigma(u(s, y)) \xi(\mathrm{d} s \mathrm{~d} y), \quad \text { and } \\
& \mathcal{T}_{4}:=\int_{(t, T) \times[0,1]} G_{T-s}(x, y) \sigma(u(s, y)) \xi(\mathrm{d} s \mathrm{~d} y) .
\end{aligned}
$$

By Lemma 3.7, $\mathcal{G}_{T} u_{0} \in \mathbb{C}_{0}^{\alpha}$ if $u_{0} \in \mathbb{C}_{0}^{\alpha}$, and $\left\|\mathcal{G}_{T} u_{0}\right\|_{\mathbb{C}_{0}^{\alpha}} \lesssim\|f\|_{\mathbb{C}_{0}^{\alpha}}$. Lemmas 3.5 and 3.7 ensure that

$$
\begin{aligned}
\sup _{x \in[0,1]}\left|\left(\mathcal{G}_{T} u_{0}\right)(x)-\left(\mathcal{G}_{t} u_{0}\right)(x)\right| & \leq \sup _{x \in[0,1]}\left|\mathcal{G}_{T-t}\left(\mathcal{G}_{t} u_{0}\right)(x)-\left(\mathcal{G}_{t} u_{0}\right)(x)\right| \\
& \lesssim\left\|\mathcal{G}_{t} u_{0}\right\|_{\mathbb{C}_{0}^{\alpha}} \cdot(T-t)^{\alpha / 2} \\
& \lesssim\left\|u_{0}\right\|_{\mathbb{C}_{0}^{\alpha}} \cdot(T-t)^{\alpha / 2}
\end{aligned}
$$

uniformly for all $u_{0} \in \mathbb{C}_{0}^{\alpha}$ and $0 \leq t<T$. Next, we estimate the $L^{k}(\Omega)$-norms of $\mathcal{T}_{1}, \ldots, \mathcal{T}_{4}$, in this order.

Lemma A.4 and inequality (3.1) together imply that

$$
\begin{aligned}
\left\|\mathcal{T}_{1}\right\|_{k} \leq & \int_{(0, t) \times(0,1)}\left|G_{T-s}(x, y)-G_{t-s}(x, y)\right|\left(c(b)+\mathrm{L}(b)\|u(s, y)\|_{k}\right) \mathrm{d} s \mathrm{~d} y \\
\lesssim & c(b)(T-t)^{1 / 2} \\
& \quad+\mathrm{L}(b) \mathrm{e}^{\beta t} \mathcal{N}_{\beta, k}(u) \int_{(0, t) \times(0,1)}\left|G_{T-s}(x, y)-G_{t-s}(x, y)\right| \mathrm{d} s \mathrm{~d} y \\
\lesssim & {\left[c(b)+\mathrm{L}(b) \mathrm{e}^{\beta t} \mathcal{N}_{\beta, k}(u)\right] \cdot(T-t)^{1 / 2}, }
\end{aligned}
$$

for all $\beta>0$. We select $\beta:=16 c^{4} \mathrm{~L}(b)$ for the same constant $c$ as was used in (3.12) to deduce from (3.12) that

$$
\left\|\mathcal{T}_{1}\right\|_{k} \leq\left[c(b)+\mathcal{M}_{3} \mathrm{~L}(b) \mathrm{e}^{16 c^{4} \mathrm{~L}(b) t}\right] \cdot(T-t)^{1 / 2},
$$

uniformly for all $x \in[0,1], 0 \leq t<T$ and $k \in\left[2, \sqrt{\mathrm{L}(b)} / \mathrm{L}(\sigma)^{2}\right]$.

Next, we bound the size of $\mathcal{T}_{2}$. In accord with (3.1) and (3.8),

$$
\begin{aligned}
\left\|\mathcal{T}_{2}\right\|_{k} & \leq \int_{(t, T) \times[0,1]} G_{T-s}(x, y)\left(c(b)+\mathrm{L}(b)\|u(s, y)\|_{k}\right) \mathrm{d} s \mathrm{~d} y \\
& \leq\left[c(b)+\mathrm{e}^{\beta T} \mathrm{~L}(b) \mathcal{N}_{\beta, k}(u)\right] \cdot(T-t),
\end{aligned}
$$

for every $\beta>0$. Once again, we choose $\beta:=16 c^{4} \mathrm{~L}(b)$ in order to see that

$$
\left\|\mathcal{T}_{2}\right\|_{k} \leq\left[c(b)+\mathcal{M}_{3} \mathrm{~L}(b) \mathrm{e}^{16 c^{4} \mathrm{~L}(b) T}\right] \cdot(T-t),
$$


uniformly for all $x \in[0,1], 0 \leq t<T$, and $k \in\left[2, \sqrt{\mathrm{L}(b)} / \mathrm{L}(\sigma)^{2}\right]$.

In order to estimate $\mathcal{T}_{3}$, we appeal to (3.1), once again, together with a suitable formulation of the BDG inequality [19], Proposition 4.4, p. 36, and deduce that

$$
\begin{aligned}
\left\|\mathcal{T}_{3}\right\|_{k}^{2} \leq & 4 k \int_{0}^{t} \mathrm{~d} s \int_{0}^{1} \mathrm{~d} y\left|G_{T-s}(x, y)-G_{t-s}(x, y)\right|^{2}\|\sigma(u(s, y))\|_{k}^{2} \\
\lesssim & k \int_{0}^{t} \mathrm{~d} s \int_{0}^{1} \mathrm{~d} y\left|G_{T-s}(x, y)-G_{t-s}(x, y)\right|^{2}\left(c(\sigma)+\mathrm{L}(\sigma)\|u(s, y)\|_{k}\right)^{2} \\
\lesssim & k c(\sigma)^{2} \cdot(T-t)^{1 / 2} \\
& +k[\mathrm{~L}(\sigma)]^{2} \cdot \int_{0}^{t} \mathrm{~d} s \int_{0}^{1} \mathrm{~d} y\left|G_{T-s}(x, y)-G_{t-s}(x, y)\right|^{2}\|u(s, y)\|_{k}^{2} ;
\end{aligned}
$$

see Lemma A.4 for the last inequality. We use, yet another time, the bound

$$
\|u(s, y)\|_{k}^{2} \leq \mathrm{e}^{2 \beta t}\left[\mathcal{N}_{\beta, k}(u)\right]^{2}
$$

(valid uniformly for all $0<s<t, y \in[0,1], k \geq 2$, and $\beta>0$ ), in order to find that

$$
\left\|\mathcal{T}_{3}\right\|_{k} \lesssim k^{1 / 2}\left[c(\sigma)+\mathrm{L}(\sigma) \mathrm{e}^{\beta t} \mathcal{N}_{\beta, k}(u)\right] \cdot(T-t)^{1 / 4} .
$$

Set $\beta:=16 c^{4} \mathrm{~L}(b)$ in order to find, as before, that because of (3.12),

$$
\left\|\mathcal{T}_{3}\right\|_{k} \lesssim k^{1 / 2}\left[c(\sigma)+\mathcal{M}_{3} \mathrm{~L}(\sigma) \mathrm{e}^{16 c^{4} \mathrm{~L}(b) t}\right] \cdot(T-t)^{1 / 4},
$$

uniformly for all $x \in[0,1], 0 \leq t<T$, and $k \in\left[2, \sqrt{\mathrm{L}(b)} / \mathrm{L}(\sigma)^{2}\right]$.

Finally, we estimate $\mathcal{T}_{4}$ by similar means: By the BDG inequality,

$$
\begin{aligned}
\left\|\mathcal{T}_{4}\right\|_{k}^{2} & \leq 4 k \int_{t}^{T} \mathrm{~d} s \int_{0}^{1} \mathrm{~d} y\left|G_{T-s}(x, y)\right|^{2}\|\sigma(u(s, y))\|_{k}^{2} \\
& \lesssim k \int_{t}^{T} \mathrm{~d} s \int_{0}^{1} \mathrm{~d} y\left|G_{T-s}(x, y)\right|^{2}\left(c(\sigma)+\mathrm{L}(\sigma)\|u(s, y)\|_{k}\right)^{2} .
\end{aligned}
$$

By Lemma A.5,

$$
\int_{t}^{T} \mathrm{~d} s \int_{0}^{1} \mathrm{~d} y\left|G_{T-s}(x, y)\right|^{2} \lesssim(T-t)^{1 / 2},
$$

uniformly for all $x \in[0,1]$ and $0 \leq t<T$. Therefore,

$$
\begin{aligned}
\left\|\mathcal{T}_{4}\right\|_{k}^{2} \lesssim & k c(\sigma)^{2} \cdot(T-t)^{1 / 2} \\
& +k[\mathrm{~L}(\sigma)]^{2} \int_{t}^{T} \mathrm{~d} s \int_{0}^{1} \mathrm{~d} y\left|G_{T-s}(x, y)\right|^{2}\|u(s, y)\|_{k}^{2} \\
\leq & k c(\sigma)^{2} \cdot(T-t)^{1 / 2} \\
& +k[\mathrm{~L}(\sigma)]^{2} \mathrm{e}^{2 \beta T}\left[\mathcal{N}_{\beta, k}(u)\right]^{2} \cdot \int_{t}^{T} \mathrm{~d} s \int_{0}^{1} \mathrm{~d} y\left|G_{T-s}(x, y)\right|^{2} \\
\leq & k\left[c(\sigma)^{2}+[\mathrm{L}(\sigma)]^{2} \mathrm{e}^{2 \beta T}\left[\mathcal{N}_{\beta, k}(u)\right]^{2}\right] \cdot(T-t)^{1 / 2},
\end{aligned}
$$


uniformly for all $\beta>0, k \geq 2,0 \leq t<T$, and $x \in[0,1]$. Once again, we select $\beta:=16 c^{4} \mathrm{~L}(b)$ and appeal to (3.12) in order to see that

$$
\left\|\mathcal{T}_{4}\right\|_{k} \lesssim k^{1 / 2}\left[c(\sigma)+\mathcal{M}_{3} \mathrm{~L}(\sigma) \mathrm{e}^{16 c^{4} \mathrm{~L}(b) T}\right] \cdot(T-t)^{1 / 4},
$$

uniformly for all $x \in[0,1], 0 \leq t<T$, and $k \in\left[2, \sqrt{\mathrm{L}(b)} / \mathrm{L}(\sigma)^{2}\right]$. Now combine displays (3.23)-(3.26) with (3.22) and (3.21) in order to see that

$$
\begin{aligned}
& \|u(T, x)-u(t, x)\|_{k} \\
& \quad \lesssim\left\|u_{0}\right\|_{\mathbb{C}_{0}^{\alpha}} \cdot(T-t)^{\alpha / 2}+k^{1 / 2}\left[\mathcal{M}_{1}+\mathcal{M}_{2} \mathcal{M}_{3} \mathrm{e}^{16 c^{4} \mathrm{~L}(b) T}\right] \cdot(T-t)^{1 / 4},
\end{aligned}
$$

uniformly for all $k \in\left[2, \sqrt{\mathrm{L}(b)} / \mathrm{L}(\sigma)^{2}\right], 0 \leq t<T$, and $x \in[0,1]$. This has the desired result; we must restrict to $0 \leq t<T \leq T_{0}$ in order to account for large values of $T-t$.

Finally, we mention the following variation of Proposition 3.11. The following includes a bound for the $k$ th moment of temporal increments of the solution to (1.1) when $k>\sqrt{\mathrm{L}(b)} / \mathrm{L}(\sigma)$.

Proposition 3.12. Fix $T_{0}>0$. Choose and fix $\alpha \in(0,1]$, and define $\mu:=$ $\min \left(\frac{1}{4}, \frac{1}{2} \alpha\right)$. If, in addition, $u_{0} \in \mathbb{C}_{0}^{\alpha}$, then

$$
\sup _{0 \leq t<T \leq T_{0}} \sup _{x \in[0,1]} \mathrm{E}\left(\left|\frac{u(T, x)-u(t, x)}{(T-t)^{\mu}}\right|^{k}\right)<\infty,
$$

for every $k \geq 2$.

ProOF. We simply adjust the proof of Proposition 3.11 by setting $\beta:=$ $c^{4} k^{2}[\mathrm{~L}(\sigma)]^{4} / 16$-instead of $\beta=16 c^{4} \mathrm{~L}(b)$-in (3.23), (3.24) and (3.25). Finally, use (3.13) instead of (3.12).

We are ready to prove Theorem 3.4.

Proof of TheOrem 3.4. Propositions 3.10 and 3.12 and a standard application of the Kolmogorov continuity theorem for random fields [20], p. 31, together imply that $u$ has a modification, which we continue to denote by $u$, that is Hölder continuous jointly in its two space-time parameters $t$ and $x$.

We note that $u(t, 0)=u(t, 1)=0$ for all $t>0$, outside a single null set. By the continuity of $t \mapsto u(t)$ - which we justified in the previous paragraph-it suffices to prove that

$$
\mathrm{P}\{u(t, 0)=u(t, 1)=0\}=1 \quad \text { for all } t>0 .
$$

Since $G_{r}(0, y)=G_{r}(1, y)=0$ for all $r>0$ and $y \in[0,1],\left(\mathcal{G}_{t} u_{0}\right)(0)=$ $\left(\mathcal{G}_{t} u_{0}\right)(1)=0$ and (3.4) implies (3.27). 
By Proposition 3.8, for all $t \geq 0$, the function $x \mapsto I(t, x)$ belongs to $\bigcap_{\varepsilon>0} \mathbb{C}_{0}^{\frac{1}{2}-\varepsilon}$ and $\mathcal{G}_{t} u_{0} \in \mathbb{C}_{0}^{\alpha}$. If $\alpha \in\left(0, \frac{1}{2}\right)$, then $\bigcap_{\varepsilon>0} \mathbb{C}_{0}^{\frac{1}{2}-\varepsilon} \subset \mathbb{C}_{0}^{\alpha}$. And whenever $\alpha \geq \frac{1}{2}$, we have $\mathbb{C}_{0}^{\alpha} \subset \bigcap_{\varepsilon>0} \mathbb{C}_{0}^{\frac{1}{2}-\varepsilon}$. This proves Theorem 3.4.

3.3. A uniform bound. The main result of this section is the following maximal inequality. It contains a locally-uniform improvement to Proposition 3.1.

THEOREM 3.13. Let $u=\{u(t, x)\}_{t \geq 0, x \in[0,1]}$ denote the continuous modification of $u$, and define $\varpi:=\max (12,6 / \alpha)$ and fix $T_{0}>0$. If $u_{0} \in \mathbb{C}_{0}^{\alpha}$ for some $\alpha \in(0,1]$ and $\sqrt{L(b)}>\varpi L(\sigma)^{2}$, then there exists a finite constant $A-$ independent of $L(b), L(\sigma)$-such that for all $T \in\left[0, T_{0}\right]$,

$$
\begin{aligned}
& \mathrm{E}\left(\sup _{t \in[0, T]} \sup _{x \in[0,1]}|u(t, x)|^{k}\right) \\
& \quad \leq A^{k}(1 \vee T)^{k\left(1+\frac{\alpha}{2} \wedge \frac{1}{4}\right)}\left(\left\|u_{0}\right\|_{\mathbb{C}_{0}^{\alpha}}^{k}+k^{k / 2} \mathcal{M}_{1}^{k}+k^{k / 2} \mathcal{M}_{2}^{k} \mathcal{M}_{3}^{k} \mathrm{e}^{A k L(b) T}\right),
\end{aligned}
$$

uniformly for all $k \in\left(\varpi, \sqrt{L(b)} / L(\sigma)^{2}\right]$.

The proof of Theorem 3.13 requires a quantitative formulation of a celebrated inequality of Garsia [15] (see also Garsia and Rodemich [16]), developed by Dalang et al. [9], Proposition A.1. First, let us recall that a function $\Psi: \mathbb{R} \rightarrow \mathbb{R}_{+}$ is a strong Young function if it is even and convex on $\mathbb{R}$, and strictly increasing on $\mathbb{R}_{+}$. Its inverse will be denoted by $\Psi^{-1}$.

LEMMA 3.14 (Garsia's lemma). Let $(S, \varrho)$ be a metric space, $v$ a Radon measure on $S$ and $\Psi: \mathbb{R} \rightarrow \mathbb{R}_{+}$a strong Young function that satisfies $\Psi(0)=0$ and $\lim _{|z| \rightarrow \infty} \Psi(z)=\infty$. Suppose $p:[0, \infty) \rightarrow \mathbb{R}_{+}$is a continuous, strictly increasing function that satisfies $p(0)=0$, and choose a continuous function $f: S \rightarrow \mathbb{R}$. Then, for every compact set $K \subset S$ and for all real numbers $\delta>0$,

$$
\sup _{\substack{a, b \in K: \\ \varrho(a, b) \leq \delta}}|f(a)-f(b)| \leq 10 \sup _{w \in K} \int_{0}^{2 \delta} \Psi^{-1}\left(\frac{\mathcal{C}}{\left|v\left(B_{\varrho}(w, u / 4)\right)\right|^{2}}\right) \mathrm{d} p(u),
$$

where $\Psi^{-1}(\infty):=\infty, B_{\varrho}(w, r):=\{z \in S: \varrho(z, w)<r\}$ for all $w \in S$ and $r>0$, and

$$
\mathcal{C}:=\int v(\mathrm{~d} a) \int v(\mathrm{~d} b) \Psi\left(\frac{f(a)-f(b)}{p(\varrho(a, b))}\right) .
$$

ProOF of TheOrem 3.13. Throughout the proof, set $\eta=\alpha \wedge \frac{1}{2}$ and $\mu:=$ $\frac{1}{4} \wedge \frac{\alpha}{2}=\frac{\eta}{2}$. Let $S$ denote the space-time continuum. That is,

$$
S:=\mathbb{R}_{+} \times[0,1] .
$$


We can define a metric $\varrho$ on $S$ as follows:

$$
\varrho((s, y),(t, x)):=|s-t|^{\mu}+|x-y|^{\eta},
$$

for every $s, t \geq 0$ and $x, y \in[0,1]$. Propositions 3.8 and 3.11 together imply that there exists a finite constant $A>0$ such that

$$
\mathrm{E}\left(|u(s, y)-u(t, x)|^{k}\right) \leq A^{k}\left(\left\|u_{0}\right\|_{\mathbb{C}_{0}^{\alpha}}^{k}+k^{k / 2} \mathcal{M}_{1}^{k}+k^{k / 2} \mathcal{M}_{2}^{k} \mathcal{M}_{3}^{k} \mathrm{e}^{A k \mathrm{~L}(b)(s \vee t)}\right)
$$

$$
\times[\varrho((s, y),(t, x))]^{k},
$$

uniformly for every real number $k \in\left[2, \sqrt{\mathrm{L}(b)} / \mathrm{L}(\sigma)^{2}\right]$, all $x, y \in[0,1]$, and all $s, t \in[0, T]$. Choose and fix some

$$
\delta \in\left(\frac{\varpi}{k}, 1\right) .
$$

This is possible because we assume $k>\varpi \geq 12$. We plan to apply Garsia's lemma (Lemma 3.14) with $p(x):=x^{\delta}, \Psi(x):=|x|^{k}$, and $v:=$ the standard Lebesgue measure on

$$
K:=[0, T] \times[0,1] .
$$

The quantity $\mathcal{C}$ of Lemma 3.14 can now be evaluated as

$$
\mathcal{C}=\int_{K \times K} \frac{|u(s, y)-u(t, x)|^{k}}{[\varrho((s, y),(t, x))]^{k \delta}} \mathrm{d} s \mathrm{~d} y \mathrm{~d} t \mathrm{~d} x .
$$

We know, thanks to (3.28) and since $\delta<1$, that $\mathrm{E}[\mathcal{C}]<\infty$, and hence $\mathcal{C}<\infty$ a.s. In fact, we can deduce from (3.28) and Lemma 3.15 below that $\{\mathrm{E}[\mathcal{C}]\}^{1 / k} \leq A\left(\left\|u_{0}\right\|_{\mathbb{C}_{0}^{\alpha}}+k^{1 / 2} \mathcal{M}_{1}+k^{1 / 2} \mathcal{M}_{2} \mathcal{M}_{3} \mathrm{e}^{A T L(b)}\right)$

$$
\begin{aligned}
& \times\left[\int_{K \times K}[\varrho((s, y),(t, x))]^{k(1-\delta)} \mathrm{d} s \mathrm{~d} y \mathrm{~d} t \mathrm{~d} x\right]^{1 / k} \\
\lesssim & A\left(\left\|u_{0}\right\|_{\mathbb{C}_{0}^{\alpha}}+k^{1 / 2} \mathcal{M}_{1}+k^{1 / 2} \mathcal{M}_{2} \mathcal{M}_{3} \mathrm{e}^{A T \mathrm{~L}(b)}\right)(1 \vee T)^{(\eta(1-\delta)+3 / k) / 2}
\end{aligned}
$$

uniformly for every $k \in\left[2, \sqrt{\mathrm{L}(b)} / \mathrm{L}(\sigma)^{2}\right]$.

Next, we note that, uniformly for all $(r, y) \in S$ and $0 \leq u \leq 4$,

$$
\begin{aligned}
v\left(B_{\varrho}((r, y), u / 4)\right) & =v\left\{(t, x):|r-t|^{\mu}+|y-x|^{\eta} \leq \frac{u}{4}\right\} \\
& \asymp v\left\{(t, x):|r-t| \lesssim u^{1 / \mu} \text { and }|y-x| \lesssim u^{1 / \eta}\right\} \\
& \asymp u^{(1 / \mu)+(1 / \eta)} \\
& =u^{3 / \eta}
\end{aligned}
$$


In particular it follows that, uniformly for all $(r, y) \in S$ and $0 \leq u \leq 4$,

$$
\Psi^{-1}\left(\frac{\mathcal{C}}{\left|v\left(B_{\varrho}((r, y), u / 4)\right)\right|^{2}}\right) \asymp \frac{\mathcal{C}^{1 / k}}{u^{6 /(\eta k)}} .
$$

As mentioned in the proof of Theorem 3.4, a classical form of the Kolmogorov continuity theorem [20], p. 31, and (3.28) together imply that $(t, x) \mapsto u(t, x)$ has a continuous modification, which we again denote $u$. Therefore, we can now see from Lemma 3.14 that there exist finite and nonrandom constants $L_{1}, L_{2}$ such that

$$
\begin{aligned}
|u(s, y)-u(t, x)|^{k} & \leq L_{1}^{k} \mathcal{C}\left[\int_{0}^{2 \varrho[(s, y),(t, x)]} \frac{u^{\delta-1}}{u^{6 /(\eta k)}} \mathrm{d} u\right]^{k} \\
& \leq L_{2}^{k} \mathcal{C}[\varrho((s, y),(t, x))]^{k \delta-6 / \eta}
\end{aligned}
$$

[where we have used that $\delta>6 /(k \eta)=\varpi / k$ ], uniformly for all $(s, y),(t, x) \in K$ and $k \in\left[\varpi, \sqrt{\mathrm{L}(b)} / \mathrm{L}(\sigma)^{2}\right]$ (it might help to notice that

$$
L_{2}=\frac{L_{1} 2^{\delta-6 /(\eta k)}}{\delta-\frac{6}{k \eta}} \leq \frac{L_{1} 2^{\delta}}{\delta-\frac{3}{\eta}}
$$

since $k \geq 2$ ). In particular, (3.30) implies that there exists a finite constant $A$ such that

$$
\begin{aligned}
\mathrm{E}\left(\sup _{\substack{(s, y),(t, x) \in K: \\
(s, y) \neq(t, x)}} \frac{|u(s, y)-u(t, x)|^{k}}{[\varrho((s, y),(t, x))]^{k \delta-6 / \eta}}\right) \\
\leq A^{k}\left(\left\|u_{0}\right\|_{\mathbb{C}_{0}^{\alpha}}^{k}+k^{k / 2} \mathcal{M}_{1}^{k}\right. \\
\left.\quad+k^{k / 2} \mathcal{M}_{2}^{k} \mathcal{M}_{3}^{k} \mathrm{e}^{A k \mathrm{~L}(b) T}\right)(1 \vee T)^{(\eta k(1-\delta)+3) / 2},
\end{aligned}
$$

uniformly for all $k \in\left[\varpi, \sqrt{\mathrm{L}(b)} / \mathrm{L}(\sigma)^{2}\right]$. The triangle inequality implies that

$$
\left\|\sup _{(t, x) \in K}|u(t, x)|\right\|_{k} \leq\left\|\sup _{(t, x) \in K}|u(t, x)-u(t, 0)|\right\|_{k}+\left\|\sup _{t \in[0, T]}|u(t, 0)|\right\|_{k} .
$$

The second term vanishes [see (3.27)], and the first term is bounded above by

$$
\left\|\sup _{\substack{(t, x) \in K: \\ x \neq 0}} \frac{|u(t, x)-u(t, 0)|}{\left(|x-0|^{\eta}\right)^{\delta-6 /(k \eta)}}\right\|_{k} \leq\left\|\sup _{\substack{(s, y),(t, x) \in K: \\(s, y) \neq(t, x)}} \frac{|u(s, y)-u(t, x)|}{[\varrho((s, y),(t, x))]^{\delta-6 /(k \eta)}}\right\|_{k} .
$$

The theorem now follows from (3.31) and the fact that $\eta(1-\delta)+3 / k \leq \eta+3 / 2$.

LEMMA 3.15. Uniformly for $T>0$,

$$
\int_{K \times K}[\varrho((s, y),(t, x))]^{k(1-\delta)} \mathrm{d} s \mathrm{~d} y \mathrm{~d} t \mathrm{~d} x \lesssim(1 \vee T)^{(k \eta(1-\delta)+3) / 2} .
$$


PROOF. The left-hand side is equal to

$$
\begin{aligned}
& \int_{K \times K}\left[|s-t|^{\eta / 2}+|x-y|^{\eta}\right]^{k(1-\delta)} \mathrm{d} s \mathrm{~d} y \mathrm{~d} t \mathrm{~d} x \\
& \quad \lesssim \int_{K \times K}\left[|s-t|^{1 / 2}+|x-y|\right]^{k \eta(1-\delta)} \mathrm{d} s \mathrm{~d} y \mathrm{~d} t \mathrm{~d} x .
\end{aligned}
$$

Set $s-t=u, x-y=v$, and bound this by

$$
C \int_{0}^{T} \mathrm{~d} u \int_{0}^{1} \mathrm{~d} v\left[u^{1 / 2}+v\right]^{k \eta(1-\delta)} .
$$

Let $u=w^{2}, \mathrm{~d} u=2 w \mathrm{~d} w$, so this is bounded above by

$$
C \int_{0}^{\sqrt{T}} \mathrm{~d} w w \int_{0}^{1} \mathrm{~d} v[w+v]^{k \eta(1-\delta)} \leq C \int_{0}^{\sqrt{T}} \mathrm{~d} w \int_{0}^{1} \mathrm{~d} v[w+v]^{k \eta(1-\delta)+1} .
$$

Pass to polar coordinates in the variables $(w, v)$ to bound this by

$$
C \int_{0}^{1 \vee \sqrt{T}} d \rho \rho^{k \eta(1-\delta)+2}=\tilde{C}(1 \vee T)^{(k \eta(1-\delta)+3) / 2} .
$$

This concludes the proof.

4. Proof of Theorem 1.9. For all $N \geq 1$, let $b_{N}$ be the following truncation of the drift function:

$$
b_{N}(z):= \begin{cases}b(z) & \text { if }|z| \leq N \\ b(N) & \text { if } z>N \\ b(-N) & \text { if } z<-N .\end{cases}
$$

Let $\sigma_{N}(z)$ denote the corresponding truncation of the diffusion coefficient $\sigma$.

Consider the stochastic PDE

$$
\dot{u}_{N}(t, x)=\frac{1}{2} u_{N}^{\prime \prime}(t, x)+b_{N}\left(u_{N}(t, x)\right)+\sigma_{N}\left(u_{N}(t, x)\right) \xi(t, x)
$$

subject to $u_{N}(0)=u_{0}$ and Dirichlet boundary conditions. Since $b_{N}, \sigma_{N}$ are globally Lipschitz, standard theory [28], Chapter 3, implies that the solution $u_{N}$ exists for all time, has a continuous modification which we again denote by $u_{N}$, and is unique almost surely. Consider also the stopping times

$$
\tau_{N}:=\inf \left\{t>0: \sup _{x \in[0,1]}\left|u_{N}(t, x)\right|>N\right\}
$$

where inf $\varnothing:=\infty$.

The local property of the stochastic integral [23], Chapter 1, imply that a.s., for $N>\left\|u_{0}\right\|_{\mathbb{L}^{\infty}}$

$$
u_{N}(t, x)=u_{N+1}(t, x) \quad \text { for all } t \in\left[0, \tau_{N}\right) \text { and } x \in[0,1] .
$$


Since $u_{N}$ is well defined for all time, and is a continuous function of $(t, x)$, this proves that $\tau_{N} \leq \tau_{N+1}$ a.s. for all $N \geq\left\|u_{0}\right\|_{\mathbb{L}^{\infty}}$ and, therefore, there exists a spacetime stochastic process $u$ such that for all $N \geq\left\|u_{0}\right\|_{\mathbb{L}^{\infty}}, u(t, x)=u_{N}(t, x)$ for all $x \in[0,1]$ and $t \in\left[0, \tau_{N}\right)$.

Consider the stopping time

$$
\tau_{\infty}=\lim _{N \uparrow \infty} \tau_{N}
$$

Our aim is to show that $\tau_{\infty}=\infty$ a.s.

The continuity of $u_{N}$ implies that $\sup _{x \in[0,1]}\left|u_{N}\left(\tau_{N}, x\right)\right|=N$ almost surely. Therefore, the preceding readily implies the following.

LEMMA 4.1. If u blows up at all, then it does so continuously. More precisely,

$$
\lim _{t \nearrow \tau_{\infty}} \sup _{x \in[0,1]}|u(t, x)|=\infty \quad \text { a.s. on }\left\{\tau_{\infty}<\infty\right\} \text {. }
$$

Now we prove Theorem 1.9.

PROOF OF THEOREM 1.9. We begin with the proof of the global existence. This is divided into three steps.

Step 1. In the first two steps, we replace $b$ in (1.1) with a function $\tilde{b}$ that has the following special form: There exist two constants $\vartheta_{1}, \vartheta_{2} \in \mathbb{R}$ such that $\vartheta_{2} \neq 0$ and

$$
\tilde{b}(z)=\vartheta_{1}+\vartheta_{2}|z| \log _{+}|z| \quad \text { for all } z \in \mathbb{R},
$$

where we recall $\log _{+}(a):=\log (a \vee$ e) for all $a \geq 0$. We may assume, without of generality, that

$$
\vartheta_{2}>0 \text {. }
$$

Indeed, the case where $\vartheta_{2}<0$ is handled by making small adjustments to the ensuing argument.

Define

$$
\tilde{b}_{N}(z):=\vartheta_{1}+\vartheta_{2}(|z| \wedge N) \log _{+}(|z| \wedge N),
$$

for all $N \geq 3$. Then $\left|\tilde{b}_{N}(z)\right| \leq \vartheta_{1}+\vartheta_{2}|z|(\log N)$ so we can take

$$
\mathrm{L}\left(\tilde{b}_{N}\right)=\vartheta_{2} \log N \text {. }
$$

In particular, for every fixed integer $N \geq 3$, the following stochastic PDE is well posed for all time:

$$
\dot{U}_{N}(t, x)=\frac{1}{2} U_{N}^{\prime \prime}(t, x)+\tilde{b}_{N}\left(U_{N}(t, x)\right)+\sigma_{N}\left(U_{N}(t, x)\right) \xi(t, x),
$$

valid for all $t>0$ and $x \in[0,1]$, subject to $U_{N}(0) \equiv u_{0}$ and the homogeneous Dirichlet boundary conditions. 
We assume that $u_{0} \in \mathbb{C}_{0}^{\alpha}$, where $\alpha \in(0,1]$. Define

$$
\tau_{N}^{(1)}:=\inf \left\{t>0: \sup _{x \in[0,1]}\left|U_{N}(t, x)\right|>N\right\},
$$

where $\inf \varnothing:=\infty$. As a central part of this proof, we plan to prove that

$$
\tau_{\infty}^{(1)}:=\lim _{N \nearrow \infty} \tau_{N}^{(1)}=\infty \quad \text { a.s. }
$$

In order to justify this assertion, note that since $|\sigma(z)|=o\left(|z|(\log |z|)^{1 / 4}\right)$ by (1.2), we can choose $\mathrm{L}\left(\sigma_{N}\right)=o\left((\log N)^{1 / 4}\right)>0$. Using (4.4), we see that

$$
\sqrt{\mathrm{L}\left(\tilde{b}_{N}\right)} / \mathrm{L}\left(\sigma_{N}\right)^{2} \rightarrow \infty \quad \text { as } N \rightarrow \infty
$$

so the inequality

$$
\sqrt{\mathrm{L}\left(\tilde{b}_{N}\right)} \geq \varpi \mathrm{L}\left(\sigma_{N}\right)^{2}
$$

holds for $N$ large enough, where $\varpi:=\max (12,6 / \alpha)$. For such $N$, take $k$ slightly larger than $\varpi$. We appeal to the Chebyshev inequality to see that for every $\varepsilon \in$ $(0,1)$ and large $N$,

$$
\begin{aligned}
\mathrm{P}\left\{\tau_{N}^{(1)}<\varepsilon\right\} & =\mathrm{P}\left\{\sup _{t \in[0, \varepsilon]} \sup _{x \in[0,1]}\left|U_{N}(t, x)\right|>N\right\} \\
& \leq N^{-k} \mathrm{E}\left(\sup _{t \in[0, \varepsilon]} \sup _{x \in[0,1]}\left|U_{N}(t, x)\right|^{k}\right) .
\end{aligned}
$$

Next, we may apply (4.4) and Theorem 3.13 [recalling the formulas for $\mathcal{M}_{1}, \mathcal{M}_{2}$ and $\mathcal{M}_{3}$ in Proposition 3.8], in order to see that there exist constants $A$ and $B$ (that do not depend on $N$ ) such that

$$
\begin{aligned}
\mathrm{E}\left(\sup _{t \in[0, \varepsilon]} \sup _{x \in[0,1]}\left|U_{N}(t, x)\right|^{k}\right) & \leq A^{k}\left\|u_{0}\right\|_{\mathbb{C}_{0}^{\alpha}}^{k}(B+\log N)^{k} \mathrm{e}^{A k \vartheta_{2} \varepsilon \log N} \\
& =A^{k}\left\|u_{0}\right\|_{\mathbb{C}_{0}^{\alpha}}^{k}(B+\log N)^{k} N^{A k \vartheta_{2} \varepsilon} .
\end{aligned}
$$

In other words, we now have

$$
\mathrm{P}\left\{\tau_{N}^{(1)}<\varepsilon\right\} \leq A^{k}\left\|u_{0}\right\|_{\mathbb{C}_{0}^{\alpha}}^{k}(B+\log N)^{k} N^{k\left(A \vartheta_{2} \varepsilon-1\right)},
$$

uniformly for all integers $N$ that satisfy $(4.6)$ and $\varepsilon \in(0,1)$. Provided that $\varepsilon<$ $A^{-1} \vartheta_{2}^{-1}$, the right-hand side converges to 0 as $N \rightarrow \infty$, so (4.7) implies that $\tau_{\infty}^{(1)} \geq$ $\varepsilon$ with probability one.

This in turn proves that

$$
\tau_{\infty}^{(1)}>\tau_{0}:=\frac{1}{2} \min \left(A^{-1} \vartheta_{2}^{-1}, 1\right) \quad \text { a.s. }
$$


Step 2. The main goal of this step is to establish the conclusions of Theorem 1.9 in the special case where $b$ in (1.1) is replaced by $\tilde{b}$ from (4.3), and to establish (4.5).

Define

$$
W_{t}(\phi):=\int_{(0, t) \times(0,1)} \phi(x) \xi(\mathrm{d} s \mathrm{~d} x) \quad \text { for all } t>0 \text { and } \phi \in \mathbb{L}^{2},
$$

with $W_{0}(\phi):=0$. Then $W(\phi)$ is a Brownian motion for every $\phi \in \mathbb{L}^{2}$. Let $\mathcal{F}_{t}^{0}$ denote the $\sigma$-algebra generated by all random variables of the form $W_{s}(\phi)$, as $s$ ranges in $[0, t]$ and $\phi$ in $\mathbb{L}^{2}$. Let $\mathcal{F}_{t}$ denote the augmented, right-continuous extension of $\mathcal{F}_{t}^{0}$ to see that $\mathcal{F}:=\left\{\mathcal{F}_{t}\right\}_{t \geq 0}$ is a complete, right-continuous filtration in the sense of general theory of processes [27]. A standard argument (see, e.g., Nualart and Pardoux [24]) shows that every process $U_{N}:=\left\{U_{N}(t)\right\}_{t \geq 0}$ is a strong Markov process with respect to $\mathcal{F}$.

Recall the nonrandom time $\tau_{0} \in(0, \infty)$ from $(4.8)$, and define $\tau_{\infty}^{(2)}$ $:=\lim _{N \nearrow \infty} \tau_{N}^{(2)}$, where

$$
\tau_{N}^{(2)}:=\inf \left\{t>\tau_{0}: \sup _{x \in[0,1]}\left|U_{N}(t, x)\right|>N\right\}
$$

where inf $\varnothing:=\infty$. According to Theorem 3.4, $U_{N}\left(\tau_{0}\right)$ is almost surely an element of $\mathbb{C}_{0}^{\alpha}$ for some $\alpha \in(0,1]$. Therefore, we can condition on $\mathcal{F}_{\tau_{0}}$ and appeal to the asserted Markov property of $U_{N}$ in order to see that $\tau_{\infty}^{(2)}>2 \tau_{0}$ a.s. Now we proceed by induction in order to see that

$$
\tau_{\infty}^{(m)}>m \tau_{0} \quad \text { a.s. for all integers } m \geq 1
$$

where $\tau_{\infty}^{(m)}:=\lim _{N \nearrow \infty} \tau_{N}^{(m)}$, for

$$
\tau_{N}^{(m)}:=\inf \left\{t>(m-1) \tau_{0}: \sup _{x \in[0,1]}\left|U_{N}(t, x)\right|>N\right\},
$$

with inf $\varnothing:=\infty$. The preceding discussion reveals that $\tau_{\infty}^{(1)} \geq \tau_{\infty}^{(m)}>m \tau_{0}$ a.s. for all $m \geq 1$. Therefore, it follows from (4.9) that $\tau_{\infty}^{(1)}=\infty$ a.s. This completes the proof of (4.5).

We now define

$$
U(t, x)=U_{N}(t, x), \quad \text { for } t \in\left[0, \tau_{N}^{(1)}\right] \text { and } x \in[0,1] .
$$

This defines $U(t, x)$ for $t \in \mathbb{R}_{+}$and $x \in[0,1]$ in a coherent way since, for each integer $N, U_{N}$ satisfies

$$
\begin{aligned}
U_{N}(t, x)= & \left(\mathcal{G}_{t} u_{0}\right)(x)+\int_{(0, t) \times(0,1)} G_{t-s}(x, y) \tilde{b}_{N}\left(U_{N}(s, y)\right) \mathrm{d} s \mathrm{~d} y \\
& +\int_{(0, t) \times(0,1)} G_{t-s}(x, y) \sigma_{N}\left(U_{N}(s, y)\right) \xi(\mathrm{d} s \mathrm{~d} y) .
\end{aligned}
$$


In particular, since for $|z| \leq N, \tilde{b}_{N}(z)=\tilde{b}_{N+1}(z)$ and $\sigma_{N}(z)=\sigma_{N+1}(z)$, as in the proof of Theorem 5.3 in [4], we have $U_{N}(t, x)=U_{N+1}(t, x)$ for $t \leq \tau_{N}^{(1)}$. Therefore, on $\left\{t \leq \tau_{N}^{(1)}\right\}$,

$$
\tilde{b}_{N}\left(U_{N}(s, y)\right)=\tilde{b}(U(s, y)), \quad \text { and } \quad \sigma_{N}\left(U_{N}(t, x)\right)=\sigma(U(t, x)),
$$

so on $\left\{t \leq \tau_{N}^{(1)}\right\}$, (4.10) becomes

$$
\begin{aligned}
U(t, x)= & \left(\mathcal{G}_{t} u_{0}\right)(x)+\int_{(0, t) \times(0,1)} G_{t-s}(x, y) \tilde{b}(U(s, y)) \mathrm{d} s \mathrm{~d} y \\
& +\int_{(0, t) \times(0,1)} G_{t-s}(x, y) \sigma(U(s, y)) \xi(\mathrm{d} s \mathrm{~d} y) .
\end{aligned}
$$

Since $N$ is arbitrary, this equation is satisfied for all $t \in \mathbb{R}_{+}$. This establishes the conclusions of Theorem 1.9 in the special case where $b$ in (1.1) is replaced by $\tilde{b}$ from (4.3). We note that the stochastic integral in (4.11) is a "localized Walsh integal" in the sense of Remark 1.8.

Step 3. Now we prove the theorem in the general case where $b$ is an arbitrary locally-Lipschitz function that satisfies the growth condition $|b(z)|=O(|z| \log |z|)$ as $|z| \rightarrow \infty$.

We can find $\vartheta_{1} \in \mathbb{R}$ and $\vartheta_{2}>0$ such that

$$
b_{-}(z) \leq b(z) \leq b_{+}(z), \quad \text { for all } z \in \mathbb{R},
$$

where

$$
b_{ \pm}(z):=\vartheta_{1} \pm \vartheta_{2}|z| \log _{+}|x|, \quad \text { for all } z \in \mathbb{R} .
$$

Using Step 2, let $U_{ \pm}(t, x)$ denote the solution to (1.1), where $b$ is replaced by $b_{ \pm}$. By analogy with (4.1), let $b_{N,-}$ and $b_{N,+}$ be the truncations of $b_{-}$and $b_{+}$, respectively. Then

$$
b_{N,-}(z) \leq b_{N}(z) \leq b_{N,+}(z) .
$$

Let $u_{N}$ be the solution to (4.2), $U_{N,-}$ (resp., $U_{N,+}$ ) be the solution to (4.2) with $b_{N}$ replaced by $b_{N,-}$ (resp., $\left.b_{N,+}\right)$. According to the comparison theorem of [11], Theorem 2.1, for all $(t, x) \in \mathbb{R}_{+} \times[0,1]$,

$$
U_{N,-}(t, x) \leq u_{N}(t, x) \leq U_{N,+}(t, x) .
$$

We have shown in Step 2 that

$$
\sup _{t \in[0, T]} \sup _{x \in[0,1]}\left|U_{ \pm}(t, x)\right|<\infty, \quad \text { for all } T>0 .
$$

For any given $(t, x)$, for $N$ sufficiently large, $U_{ \pm}(t, x)=U_{N, \pm}(t, x)$, therefore (4.12) implies that

$$
U_{-}(t, x) \leq u_{N}(t, x) \leq U_{+}(t, x)
$$


Recall that

$$
\tau_{N}=\inf \left\{t>0: \sup _{x \in[0,1]}\left|u_{N}(t, x)\right|>N\right\} .
$$

Then (4.13) and (4.14) imply that $\lim _{N \rightarrow \infty} \tau_{N}=\infty$ a.s., and we can define

$$
u(t, x)=u_{N}(t, x), \quad \text { for } t \in\left[0, \tau_{N}\right] \text { and } x \in[0,1] .
$$

As above, this definition is coherent. By (4.14),

$$
U_{-}(t, x) \leq u(t, x) \leq U_{+}(t, x), \quad \text { for all } t \in \mathbb{R}_{+} \text {and } x \in[0,1] .
$$

Since

$$
\begin{aligned}
u_{N}(t, x)= & \left(\mathcal{G}_{t} u_{0}\right)(x)+\int_{(0, t) \times(0,1)} G_{t-s}(x, y) b_{N}\left(u_{N}(s, y)\right) \mathrm{d} s \mathrm{~d} y \\
& +\int_{(0, t) \times(0,1)} G_{t-s}(x, y) \sigma_{N}\left(u_{N}(s, y)\right) \xi(\mathrm{d} s \mathrm{~d} y),
\end{aligned}
$$

and on $\left\{\tau_{N}>t\right\}, b_{N}\left(u_{N}(s, y)\right)=b(u(s, y))$ and $\sigma_{N}\left(u_{N}(s, y)\right)=\sigma(u(s, y))$, the local property of the stochastic integral [23], Chapter 1, implies that on $\left\{\tau_{N}>t\right\}$,

$$
\begin{aligned}
u(t, x)= & \left(\mathcal{G}_{t} u_{0}\right)(x)+\int_{(0, t) \times(0,1)} G_{t-s}(x, y) b(u(s, y)) \mathrm{d} s \mathrm{~d} y \\
& +\int_{(0, t) \times(0,1)} G_{t-s}(x, y) \sigma(u(s, y)) \xi(\mathrm{d} s \mathrm{~d} y) .
\end{aligned}
$$

Since $\mathrm{P}\left(\bigcup_{N \in \mathbb{N}}\left\{\tau_{N}>t\right\}\right)=1$, we see that this equality is satisfied a.s. and, therefore, $u$ is a random field solution of (1.1). By (4.13) and (4.14),

$$
\sup _{t \in[0, T]} \sup _{x \in[0,1]}|u(t, x)|<\infty \quad \text { for all } T>0 .
$$

This establishes the existence statement in Theorem 1.9 as well as (1.3). The solution $U$ is continuous by (4.15), since each $U_{N}$ is continuous.

Finally, we establish uniqueness of the solution to (1.1). Let $v=\{v(t, x)\}$ be a solution of (1.1) (with initial condition $\left.u_{0}\right)$ in $C\left(\mathbb{R}_{+} \times[0,1]\right)$. We will show that $v=u$, where $u$ was constructed in Step 3 above. Define

$$
\tau_{N}^{(1)}(v)=\inf \left\{t \geq 0: \sup _{x \in[0,1]}|v(t, x)|>N\right\} .
$$

By sample path continuity of $v, \tau_{N}^{(1)}(v)>0$ a.s., and $\lim _{N \rightarrow+\infty} \tau_{N}^{(1)}(v)=+\infty$ a.s. On $\left[0, \tau_{N}^{(1)}(v)\right], v$ solves (4.2). Since $b_{N}$ and $\sigma_{N}$ are globally Lipschitz, the standard uniqueness statement implies that for all $t \geq 0$,

$$
v\left(t \wedge \tau_{N}^{(1)}(v) \wedge \tau_{N}^{(1)}(u)\right)=u\left(t \wedge \tau_{N}^{(1)}(v) \wedge \tau_{N}^{(1)}(u)\right) .
$$

We let $N \rightarrow+\infty$. By (4.5), $\tau_{N}^{(1)}(u) \rightarrow+\infty$ a.s., so we deduce that $v(t)=u(t)$, for all $t \geq 0$. This completes the proof of Theorem 1.9. 


\section{APPENDIX: ON THE GREEN'S FUNCTION}

Let us solve $\dot{u}=\frac{1}{2} u^{\prime \prime}$ in $[0,1]$ subject to the initial condition $u_{0}:=\delta_{y}$ and boundary conditions $u_{t}(0)=u_{t}(1)=0$ for all $t>0$. This endeavor yields the formula (2.5) for the fundamental solution, which we denote by $G_{t}(x, y)$. In accord with the maximum principle, $G_{t}(x, y) \geq 0$ for all $t>0$ and $x, y \in[0,1]$.

Our next results are definitely well known, as well as simple. But we include them since we will need to know about the parameter dependencies.

LEMMA A.1. Uniformly for all $w \geq v>0$,

$$
\sum_{n=1}^{\infty}\left(w+v n^{2}\right)^{-1} \lesssim \frac{1}{\sqrt{v w}}
$$

PROOF. We can bound the preceding sum from above by $S_{1}+S_{2}$, where

$$
\begin{aligned}
& S_{1}:=\sum_{n=1}^{\lfloor\sqrt{w / v}\rfloor} w^{-1} \leq(v w)^{-1 / 2}, \quad \text { and } \\
& S_{2}:=v^{-1} \sum_{n=1+\lfloor\sqrt{w / v}\rfloor}^{\infty} n^{-2} \leq v^{-1} \int_{\sqrt{w / v}}^{\infty} z^{-2} \mathrm{~d} z \leq(v w)^{-1 / 2} .
\end{aligned}
$$

The lemma follows from these inequalities.

LEMMA A.2. Uniformly for every $\beta>0$ and $\theta \in\{1,2\}$,

$$
\int_{0}^{\infty} \mathrm{d} t \int_{0}^{1} \mathrm{~d} y \mathrm{e}^{-\beta t}\left|G_{t}(x, y)\right|^{\theta} \lesssim \beta^{-1 / \theta}
$$

Proof. The case $\theta=1$ follows simply because $\int_{0}^{1} G_{t}(x, y) \mathrm{d} y \leq 1$. that

Also, the fact that $\int_{0}^{1}\left[G_{t}(x, y)\right]^{2} \mathrm{~d} y \leq 2 \sum_{n=1}^{\infty} \exp \left(-n^{2} \pi^{2} t\right)$ [by (2.5)] implies

$$
\int_{0}^{\infty} \mathrm{d} t \int_{0}^{1} \mathrm{~d} y \mathrm{e}^{-\beta t}\left[G_{t}(x, y)\right]^{2} \leq 2 \sum_{n=1}^{\infty} \int_{0}^{\infty} \mathrm{e}^{-\beta t-n^{2} \pi^{2} t} \mathrm{~d} t \leq 2 \sum_{n=1}^{\infty}\left(\beta+n^{2} \pi^{2}\right)^{-1} .
$$

The result follows from Lemma A.1.

LEMMA A.3. If $\theta \in\{1,2\}$, then

$$
\int_{0}^{\infty} \mathrm{d} t \int_{0}^{1} \mathrm{~d} y\left|G_{t}(x, y)-G_{t}\left(x^{\prime}, y\right)\right|^{\theta} \lesssim \Psi_{\theta}\left(\left|x-x^{\prime}\right|\right)
$$

uniformly for all $x, x^{\prime} \in[0,1]$, where $\Psi_{1}(z):=z \log \left(\mathrm{e} \vee z^{-1}\right)$ and $\Psi_{2}(z):=z$ for all $z>0$. 
ProOF. First, let us consider the case that $\theta=2$ and $\left|x-x^{\prime}\right|<\mathrm{e}^{-1}$. We may apply (2.5) to find that

$$
\begin{aligned}
\int_{0}^{1}\left[G_{t}(x, y)-G_{t}\left(x^{\prime}, y\right)\right]^{2} \mathrm{~d} y & =2 \sum_{n=1}^{\infty}\left[\sin (n \pi x)-\sin \left(n \pi x^{\prime}\right)\right]^{2} \mathrm{e}^{-n^{2} \pi^{2} t} \\
& \leq 2 \pi^{2} \sum_{n=1}^{\infty} \min \left(1, n^{2}\left|x-x^{\prime}\right|^{2}\right) \mathrm{e}^{-n^{2} \pi^{2} t}
\end{aligned}
$$

Therefore,

$$
\int_{0}^{\infty} \mathrm{d} t \int_{0}^{1} \mathrm{~d} y\left[G_{t}(x, y)-G_{t}\left(x^{\prime}, y\right)\right]^{2} \lesssim \sum_{n=1}^{\infty} \min \left(\frac{1}{n^{2}},\left|x-x^{\prime}\right|^{2}\right) .
$$

Since $\min \left(r^{-2}, R\right) \leq 2\left(r^{2}+R^{-1}\right)^{-1}$ for every $r, R>0$, Lemma A. 1 completes the proof in the case that $\theta=2$.

If $\theta=1$, then we likewise have

$$
\begin{aligned}
\int_{0}^{1}\left|G_{t}(x, y)-G_{t}\left(x^{\prime}, y\right)\right| \mathrm{d} y & \lesssim \sum_{n=1}^{\infty}\left|\sin (n \pi x)-\sin \left(n \pi x^{\prime}\right)\right| \mathrm{e}^{-n^{2} \pi^{2} t / 2} \\
& \lesssim \sum_{n=1}^{\infty} \min \left(1, n\left|x^{\prime}-x\right|\right) \mathrm{e}^{-n^{2} \pi^{2} t / 2} .
\end{aligned}
$$

Therefore,

$$
\begin{aligned}
\int_{0}^{\infty} & \mathrm{d} t \int_{0}^{1} \mathrm{~d} y\left|G_{t}(x, y)-G_{t}\left(x^{\prime}, y\right)\right| \\
& \lesssim \sum_{n=1}^{\infty} \frac{\min \left(1, n\left|x^{\prime}-x\right|\right)}{n^{2}} \\
& \lesssim\left|x^{\prime}-x\right| \sum_{n=1}^{\left\lfloor 1 /\left|x^{\prime}-x\right|\right\rfloor} n^{-1}+\sum_{n=1+\left\lfloor 1 /\left|x^{\prime}-x\right|\right\rfloor}^{\infty} n^{-2},
\end{aligned}
$$

and the result follows if $\left|x^{\prime}-x\right| \leq \mathrm{e}^{-1}$. If $\left|x^{\prime}-x\right|>\mathrm{e}^{-1}$, then the very same estimates show that

$$
\int_{0}^{\infty} \mathrm{d} t \int_{0}^{1} \mathrm{~d} y\left|G_{t}(x, y)-G_{t}\left(x^{\prime}, y\right)\right| \lesssim \sum_{n=1}^{\infty} n^{-2} \lesssim\left|x^{\prime}-x\right|,
$$

and this completes the proof.

LEMMA A.4. Uniformly for every $\varepsilon>0$ and $\theta \in\{1,2\}$,

$$
\sup _{t>0} \sup _{x \in[0,1]} \int_{0}^{t} \mathrm{~d} s \int_{0}^{1} \mathrm{~d} y\left|G_{t+\varepsilon-s}(x, y)-G_{t-s}(x, y)\right|^{\theta} \lesssim \sqrt{\varepsilon} .
$$


ProOF. We first consider the case that $\theta=1$. We can bound $|\sin (\ldots)|$ from above by 1 in (2.5) in order to find that the left-hand side is at most

$$
2 \sum_{n=1}^{\infty}\left[1-\mathrm{e}^{-n^{2} \pi^{2} \varepsilon / 2}\right] \int_{0}^{t} \mathrm{e}^{-n^{2} \pi^{2}(t-s) / 2} \mathrm{~d} s \lesssim \sum_{n=1}^{\infty} \min \left(n^{-2}, \varepsilon\right),
$$

since $1-\mathrm{e}^{-\theta} \leq \min (1, \theta)$ for every $\theta \geq 0$. If $\varepsilon<1$, then a direct calculation as in the proof of Lemma A.3 shows that the series is bounded by const $\cdot \sqrt{\varepsilon}$. If $\varepsilon \geq 1$, then the series is a constant $c$, which is $\leq c \sqrt{\varepsilon}$. The result for $\theta=1$ follows.

For $\theta=2$, by (2.5), the left-hand side is equal to

$$
\begin{aligned}
4 \int_{0}^{t} \sin ^{2}(n \pi x)\left(\mathrm{e}^{-n^{2} \pi^{2}(t+\varepsilon-s) / 2}-\mathrm{e}^{-n^{2} \pi^{2}(t-s) / 2}\right)^{2} \mathrm{~d} s \\
\quad \leq 4 \sum_{n=1}^{\infty}\left(1-\mathrm{e}^{-n^{2} \pi^{2} \varepsilon / 2}\right)^{2} \int_{0}^{t} \mathrm{e}^{-n^{2} \pi^{2}(t-s)} \mathrm{d} s \\
\quad \lesssim \sum_{n=1}^{\infty} \min \left(n^{-2}, n^{2} \varepsilon^{2}\right) .
\end{aligned}
$$

Then we proceed as we did when $\theta=1$.

LEMMA A.5. For all $\varepsilon>0$ and $\theta \in\{1,2\}$,

$$
\sup _{t \geq 0} \sup _{x \in[0,1]} \int_{t}^{t+\varepsilon} \mathrm{d} s \int_{0}^{1} \mathrm{~d} y\left|G_{t+\varepsilon-s}(x, y)\right|^{\theta} \lesssim \varepsilon^{1 / \theta} .
$$

Proof. For $\theta=1$, we appeal to (2.5) and (2.6), to see that $\int_{0}^{1} G_{t+\varepsilon-s}(x$, y) $\mathrm{d} y \leq 1$, and this proves the desired inequality.

For $\theta=2$, we appeal to (2.5) using that

$$
\int_{0}^{1}\left[G_{t}(x, y)\right]^{2} \mathrm{~d} y=4 \sum_{n=1}^{\infty} \sin ^{2}(n \pi x) \exp \left(-n^{2} \pi^{2} t\right),
$$

to see that

$$
\begin{aligned}
\sup _{t>0} \sup _{x \in[0,1]} \int_{t}^{t+\varepsilon} \mathrm{d} s \int_{0}^{1} \mathrm{~d} y\left|G_{t+\varepsilon-s}(x, y)\right|^{2} & \lesssim \sum_{n=1}^{\infty} \int_{0}^{\varepsilon} \mathrm{e}^{-n^{2} \pi^{2} s} \mathrm{~d} s \\
& \lesssim \sum_{n=1}^{\infty}\left(\frac{1-\mathrm{e}^{-n^{2} \pi^{2} \varepsilon / 2}}{n^{2}}\right),
\end{aligned}
$$

which is at most a constant multiple of $\sum_{n=1}^{\infty} \min \left(n^{-2}, \varepsilon\right) \lesssim \varepsilon^{1 / 2}$. This proves the result in the case that $\theta=2$. 


\section{REFERENCES}

[1] Bally, V., Millet, A. and Sanz-Solé, M. (1995). Approximation and support theorem in Hölder norm for parabolic stochastic partial differential equations. Ann. Probab. 23 178-222. MR1330767

[2] Bass, R. F. (1998). Diffusions and Elliptic Operators. Probability and Its Applications (New York). Springer, New York. MR1483890

[3] Bonder, J. F. and Groisman, P. (2009). Time-space white noise eliminates global solutions in reaction-diffusion equations. Phys. D 238 209-215. MR2516340

[4] Cerrai, S. (2003). Stochastic reaction-diffusion systems with multiplicative noise and nonLipschitz reaction term. Probab. Theory Related Fields 125 271-304. MR1961346

[5] Cerrai, S. (2011). Averaging principle for systems of reaction-diffusion equations with polynomial nonlinearities perturbed by multiplicative noise. SIAM J. Math. Anal. $432482-$ 2518. MR2854919

[6] Chen, L. and Dalang, R. C. (2014). Hölder-continuity for the nonlinear stochastic heat equation with rough initial conditions. Stoch. Partial Differ. Equ. Anal. Comput. 2 316352. MR3255231

[7] Dalang, R., Khoshnevisan, D., Mueller, C., Nualart, D. and XiaO, Y. (2009). A Minicourse on Stochastic Partial Differential Equations. Lecture Notes in Math. 1962. Springer, Berlin. MR1500166

[8] DALANG, R. C. (1999). Extending the martingale measure stochastic integral with applications to spatially homogeneous s.p.d.e.'s. Electron. J. Probab. 4 Article ID 6. MR1684157

[9] Dalang, R. C., Khoshnevisan, D. and Nualart, E. (2007). Hitting probabilities for systems of non-linear stochastic heat equations with additive noise. ALEA Lat. Am. J. Probab. Math. Stat. 3 231-271. MR2365643

[10] Dalang, R. C., Khoshnevisan, D. and Nualart, E. (2009). Hitting probabilities for systems for non-linear stochastic heat equations with multiplicative noise. Probab. Theory Related Fields 144 371-427. MR2496438

[11] Donati-Martin, C. and Pardoux, É. (1993). White noise driven SPDEs with reflection. Probab. Theory Related Fields 95 1-24. MR1207304

[12] FANG, S. and Zhang, T. (2005). A study of a class of stochastic differential equations with non-Lipschitzian coefficients. Probab. Theory Related Fields 132 356-390. MR2197106

[13] Foondun, M. and Khoshnevisan, D. (2009). Intermittence and nonlinear parabolic stochastic partial differential equations. Electron. J. Probab. 14 548-568. MR2480553

[14] Galaktionov, V. A. and VázQuez, J. L. (2002). The problem of blow-up in nonlinear parabolic equations. Discrete Contin. Dyn. Syst. 8 399-433. Current developments in partial differential equations (Temuco, 1999). MR1897690

[15] Garsia, A. M. (1972). Continuity properties of Gaussian processes with multidimensional time parameter. In Proc. Sixth Berkeley Symp. on Math. Statist. and Probab. Vol. II: Probability Theory 369-374. Univ. California Press, Berkeley, CA. MR0410880

[16] Garsia, A. M. and Rodemich, E. (1974). Monotonicity of certain functionals under rearrangement. Ann. Inst. Fourier (Grenoble) 24 67-116. Colloque International sur les Processus Gaussiens et les Distributions Aléatoires (Colloque Internat. du CNRS, No. 222, Strasbourg, 1973). MR0414802

[17] Gross, L. (1993). Logarithmic Sobolev inequalities and contractivity properties of semigroups. In Dirichlet Forms (Varenna, 1992). Lecture Notes in Math. 1563 54-88. Springer, Berlin. MR1292277

[18] GYÖNGY, I. and RoviRA, C. (2000). On $L^{p}$-solutions of semilinear stochastic partial differential equations. Stochastic Process. Appl. 90 83-108. MR1787126

[19] Khoshnevisan, D. (2014). Analysis of Stochastic Partial Differential Equations. CBMS Regional Conference Series in Mathematics 119. Amer. Math. Soc., Providence, RI. MR3222416 
[20] Kunita, H. (1990). Stochastic Flows and Stochastic Differential Equations. Cambridge Studies in Advanced Mathematics 24. Cambridge Univ. Press, Cambridge. MR1070361

[21] LiU, W. and Röckner, M. (2015). Stochastic Partial Differential Equations: An Introduction. Universitext. Springer, Cham. MR3410409

[22] Mueller, C. (1991). On the support of solutions to the heat equation with noise. Stoch. Stoch. Rep. 37 225-245. MR1149348

[23] Nualart, D. (2006). The Malliavin Calculus and Related Topics, 2nd ed. Probability and Its Applications (New York). Springer, Berlin. MR2200233

[24] Nualart, D. and Pardoux, E. (1994). Markov field properties of solutions of white noise driven quasi-linear parabolic PDEs. Stoch. Stoch. Rep. 48 17-44. MR1786190

[25] Pardoux, E. (1979). Stochastic partial differential equations and filtering of diffusion processes. Stochastics 3 127-167. MR0553909

[26] Samarskit, A. A., Galaktionov, V. A., Kurdyumov, S. P. and Mikhailov, A. P. (1995). Blow-up in Quasilinear Parabolic Equations. De Gruyter Expositions in Mathematics 19. de Gruyter, Berlin. Translated from the 1987 Russian original by Michael Grinfeld and revised by the authors. MR1330922

[27] Sharpe, M. (1988). General Theory of Markov Processes. Pure and Applied Mathematics 133. Academic Press, Boston, MA. MR0958914

[28] Walsh, J. B. (1986). An introduction to stochastic partial differential equations. In École D'été de Probabilités de Saint-Flour XIV-1984. Lecture Notes in Math. 1180 265-439. Springer, Berlin. MR0876085

R. C. DALANG

INSTITUT DE MATHÉMATIQUES

ECOLE PolytechniQue FÉdÉRALE de LAUSANNE

STATION 8

CH-1015 LAUSANNE

SWITZERLAND

E-MAIL: robert.dalang@epfl.ch
D. KHOSHNEVISAN

DEPARTMENT OF MATHEMATICS

UNIVERSITY OF UTAH

155 S. 1400 E.

SALT LAKE City, UTAH 84112-0090

USA

E-MAIL: davar@math.utah.edu

T. ZHANG

SCHOOL OF MATHEMATICS

UNIVERSITY OF MANCHESTER

OXFORD ROAD, MANCHESTER M13 9PL

UNITED KINGDOM

E-MAIL: Tusheng.Zhang@manchester.ac.uk 